\title{
An Empirical Comparison of Monitoring Algorithms for Access Anomaly
} Detection

\author{
hy'
}

Inne Dinning. Edith Schomberg

$$
\text { I litracomputer Note \#160 }
$$

December, 1989

This work was upported in part hy an IBM Ciraduate I cllowship and in part by the Ipplicd Mathemalle.l

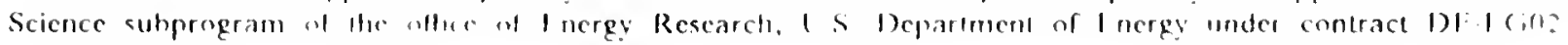
$881: R 25052$. 



\section{An Empirical Comparison of Monitoring Algorithms for Access Anomaly Detection}

\author{
Anne Dinning * \\ Courant Institute of Mathematical Sciences \\ New York University \\ 251 Mercer Street, New York, NY 10012 \\ (dinning@cs.nyu.edu)
}

\author{
Edith Schonberg ${ }^{\dagger}$ \\ IB.M T.J. Watson Research Center \\ P.O. Box 218 \\ Yorktown Heights, NY 10598 \\ (schnbrg@ibm.com)
}

\begin{abstract}
One of the major disadvantages of paralJel programming with shared memory is the nondeterministic behavior caused by uncoordinated access to shared variables, known as access anomalies. Monitoring program execution to detect access anomalies is a promising and relatively unexplored approach to this problem. We present a new algorithm, referred to as task recycling, for detecting anomalies, and compare it to an existing algorithm. Empirical results indicate several significant conclusions: (i) While space requirements are bounded by $\mathrm{O}(T \times V)$, where $T$ is the maximum number of threads that may potentially execute in parallel and $V$ is the number of variable monitored, for typical programs space requirements are on average $\mathrm{O}(V)$. (ii) Task recycling is more efficient in terms of space requirements and often in performance. (iii) The general approach of monitoring to detect access anomalies is practical.
\end{abstract}

\section{Introduction}

Erroneous non-deterministic behavior in shared memory parallel programs is often the result of access

\footnotetext{
- This research was supported in part by an IBM Graduate Fellowship.

'This research was supported in part by the Applied Mathematical Science subprogram of the office of Energy Research. U.S. Department of Energy under contract number DE-FGO288ER25052.
}

anomalies. An access anomaly occurs when two concurrent execution threads access the same memory location in an "unsafe" manner: more specifically, when either two concurrent threads both write or one reads and one writes a shared memory location without coordinating these accesses. The program segment in Figure 1 illustrates an access anomaly. The doall construct creates two parallel threads that both write the variable. $Y$. The

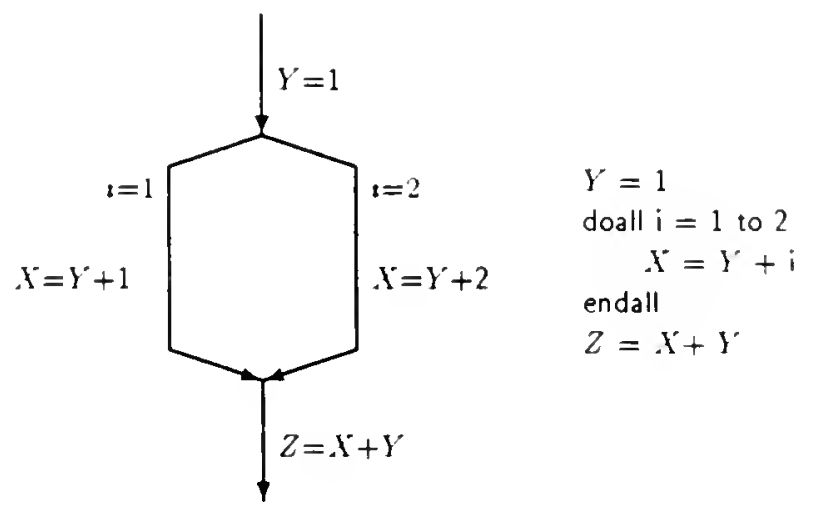

Figure 1: Simple Program With an Access Anomaly

value of $X$ subsequently used to calculate $Z$ depends on whether iterate $i=1$ or $i=2$ updates $X$ last. Therefore the two writes to $X$ are anomalous. Variable ${ }^{\circ}$ is also accessed in both concurrent threads $i=1$ and $1=2$; however, this is not an anomaly because both accesses are reads. Similarly, the assignment to $Y$ in the first statement does not cause an anomaly because it is always performed before either read. While certain in. ternal non-determanism [5] does not affect the outcome of programs and is safe (e.g. the non-deterministic order in which a lock is granted), access anomalies often introduce erternal non-determunusm that modifies execulton results. Although some programs are designed to contan access anomalies. this type of external nondetermunism is usually unintentional. Traditional debugging techniques are of limited use in finding errors 

caused by access anomalies because such bugs are sensitive to timing.

Detecting access anomalies by monttoring program execution is proposed in $[12,10,11,4]$. Using this approach, access anomaly are detected much in the same manner that array subscript range checking is performed. When a variable is accessed during execution, an immediate check is make to see whether the access conflicts with a previous access, in which case the error is reported. This approach may be used in conjunction with static analysis [5, 2] and is much more efficient that trace-based post-mortem methods [1, 3, 8].

Thus paper describes a new on-the-fly access anomaly algorithm called task recychng and compares it to an existing method. English-Hebrew labeling [9, 10]. These methods were implemented in the parallel Fortran compiler on the NYU Ultracomputer [6]. Both algorithms record read and write events in access histories that are associated with monitored variables. While in the worst case the length of an access history depends on the amount of parallelism in the program, experimental results show that access histories in fact rarely have more than a few entries ${ }^{2}$.

The two algorithms differ in the methods used to determine whether different threads are concurrent. The experiment shows that task recycling is less efficient for maintaining concurrency information at parallel operations than English-Hebrew labeling. However, it is more efficient when checking for anomalies at read and write events and requires considerably less storage. Since read and write events are generally more frequent than parallel operations, we conclude that task recycling is an improvement over English-Hebrew labeling both in performance and space.

Finally, the experiment measures the actual cost of monitoring. For the benchmark programs, monitoring entails a 3 -fold to 6 -fold slowdown. Although this cost is high, it is not unreasonable during a debugging phase of program development. Moreover, this is the cost of monitoring every shared variable reference. In general, static analysis or user directives may be used to limit the number of variables monitored, and thus greatly reduce the cost. Section 2 states the anomaly detection problem in terms of the partial order execution graph and describes access histories. The two algorithms for determining concurrency relationships are presented in Sections 3 and 4 . The experiment is presented in Section 5, and the results summarized in Section 6.

\footnotetext{
${ }^{1}$ This algorithm relies of version numbers and parent vectors. which are similarly used in [3] for post-mortem analysis.

${ }^{2}$ Access history based methods are therefore superior to the method described in $[11]$ in which the size of the access data depends on the parallelism
}

\section{Definitions and Framework}

The Ultracomputer parallel Fortran compiler provides the nestable doall-endall construct as its primary concurrency primitive. Concurrent threads are created by a doall operation and terminated by a corresponding endall operation. Coordination primitives provided as library routines may be classified as synchronous or asynchronous ${ }^{3}$. If two threads coordinate synchronously (e.g. via a barrier operation) neither thread may execute beyond the coordination point until both have reached it. If two threads coordinate asynchronously (e.g. via a signal operation) the receiver may not execute beyond the coordination point until the sender has reached it; however, the sender may proceed immediately. A block is an instruction sequence, executed by a single thread, that does not include doall, endall or coordination operations. Hence a thread is made up of a sequence of blocks that reflect its interaction with other threads.

The concurrency relationship among blocks is represented by a directed partial order execution graph. (POEG). A POEG captures Lamport's "happens before" relation and imposes a partial order on the set of blocks that make up an execution instance [7]. An edge is either a block or coordination edge: a coordination edge connects vertices of two coordinating blocks. A vertex corresponds to a doall, endall or coordination operation. Concurrency determination is independent of the number and relative execution speeds of processors executing the program. A block $b_{j}$ is an ancestor of a block $b_{i}$ if there is a path from $b_{j}$ to $b_{i}$ in the graph (equivalently, $b_{i}$ is a descendant of $b_{j}$ ). Two blocks are concurrent iff neither is an ancestor of the other. We define the maximum concurrency of a POEG to be the maximum number of mutually concurrent blocks. To illustrate these definitions, consider the POEG in Figure 2. Block $b_{3}$ is concurrent with blocks $b_{4}, b_{5}$ and $b_{2}$; it is not concurrent with block $b_{0}$ and $b_{1}$ (which are ancestors) or with blocks $b_{6}-b_{13}$ (which are descendants) The maximum concurrency of the graph is four.

In access history based algorithms, each block has an associated label. The access history for a variable. is a set of labels of the blocks which have read and written $X$. Every time variable $X$ is read or written, the access history is examined to determine whether the current event conflicts with a previous one. When block $b$ reads $\mathcal{X}$, it nust be determined whether $b$ is concurrent with the writers in the access history for,$X$. When block $b$ writes,$i$, tests are performed to determine whether $b$ is concurrent with any of the blocks in the access history of $x$. Therefore, the efficiency of an access anomaly al-

\footnotetext{
${ }^{3}$ We assume that all interthread coordination is explicit: we do not attempt to solve the problem of automatically detecting coordination
} 



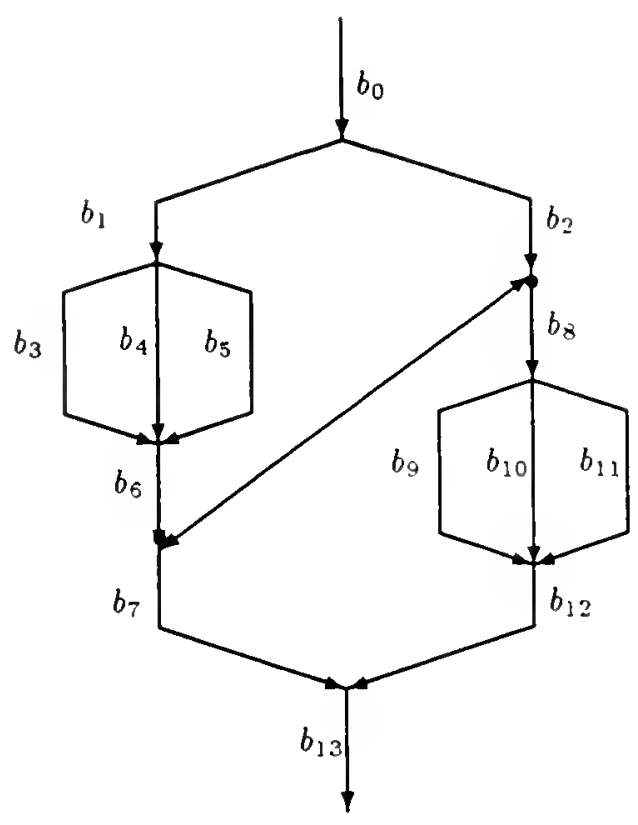

Figure 2: Partial Order Execution Graph

gorithm depends primarily on how quickly the test of concurrency can be made and how many entries are in the access history. We next show how access histories can be compressed.

Consider the accesses to variable $X$ in the POEG in Figure 3. The write of $X$ in $b_{9}$ conflicts with the reads of $X$ in $b_{2}$ and $b_{6}$. Suppose $X$ is read in $b_{2}, b_{6}$, and $b_{3}$ in that order, and then written in $b_{9}$. After the first read, the access history of $X$ contains $b_{2}$. When $X$ is next read in $b_{6}, b_{6}$ is added to the access history and $b_{2}$ is deleted. The $b_{2}$ read event is no longer needed: any subsequent write event that conflicts with $b_{6}$ also conflicts with $b_{2}$. On the other hand, when $b_{3}$ is added to the access history, $b_{6}$ cannot be deleted. Otherwise, no anomaly can be detected when $X$ is written in $b_{9}$, since this write does not conflict with the read in $b_{3}$.

More generally, a block $b$ in the reader set of a variable $X$ can be deleted when a descendant of $b$ accesses $X$. Thus, the reader set of an access history contains two blocks only if they are concurrent. (On the other hand, since two concurrent writes always conflict, there is at most one writer in an access history.) By deleting obsolete entries, the size of an access history is therefore bounded by the maximum concurrency of the POEG. We show in Section 5 that this bound is, in fact, much too pessimistic. Although compaction reduces the size of the reader and writer sets, certain information may be lost. In particular, if there are multiple anomalies involving the same variable some of them may not be reported. However, at least one anomaly is guaranteed to be reported for every variable which is accessed in an

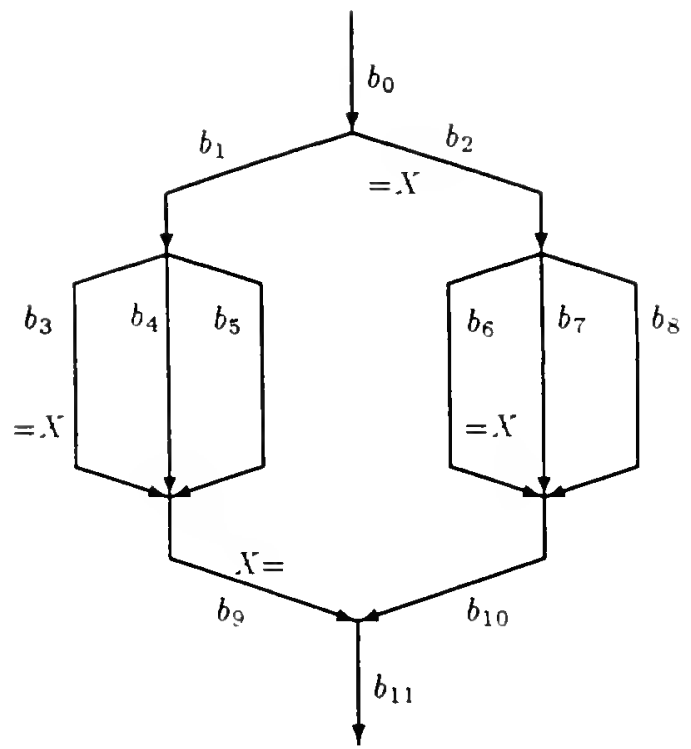

Figure 3: A Partial Order Execution Graph with Access Anomalies

"unsafe" manner.

\section{English-Hebrew Labeling}

In the English-Hebrew labeling algorithm, the structure of the POEG - and hence, the concurrency relationship among blocks - is encoded in tags $[10,9]$ associated with blocks. A tag consists of a pair of labels: an Englash label $E$ and a Hebrew label $H$. Conceptually, the English label is produced by performing a left-to-right preorder numbering of the POEG; each block is assigned a number less than all of the numbers assigned to its children and its siblings to the right. However, since this label must be generated on-line, a complete traversal of the execution graph cannot be performed. Therefore, a label is a string of numbers and labels are lextcographically ordered. The children blocks $c_{0} \ldots c_{m}$ of a doall vertex with parent block $p$ are assigned English labels:

$$
\text { doall: } E\left(\operatorname{tag}_{c_{1}}\right)-E\left(\operatorname{tag}_{p}\right) \mid i
$$

where $\mid$ is the append operation. Similarly, a block $c$ created in a coordination operation which has parent $p$ is assigned an English label:

$$
\text { coordination: } E\left(\operatorname{tag}_{c}\right)-E\left(\operatorname{tag}_{p}\right) \mid 1
$$

The child block $c$ of an endall vertex with parents $p_{0}$ $\ldots p_{m}$ is assigned English label:

$$
\text { endall } E\left(\operatorname{tag}_{c}\right)-\max \left(E\left(\operatorname{tag}_{\mathfrak{r}_{0}}\right)\right)
$$

The llebrew labels are created symmetricly for a rightto-left ordering; the only difference is that the $i^{\text {th }}$ child of a doall operation has $f+I-i$ as the last entry in its Hebrew tag. As specified above, the length of the labels 

increase with the number of doall and coordination operations. However, an additional heuristic described in [10] bounds the label length to the level of nesting.

Two tags $\operatorname{tag}_{i}$ and $\operatorname{tag}_{j}$ are unordered iff either of the following conditions is met :

$$
\begin{aligned}
& E\left(\operatorname{tag}_{i}\right)<E\left(\operatorname{tag}_{j}\right) \text { and } H\left(\operatorname{tag}_{i}\right)>H\left(\operatorname{tag}_{j}\right) \text { or } \\
& E\left(\operatorname{tag}_{i}\right)>E\left(\operatorname{tag}_{j}\right) \text { and } H\left(\operatorname{tag}_{i}\right)<H\left(\operatorname{tag}_{j}\right)
\end{aligned}
$$

English-Hebrew tags only encode the ordering due to doall and endall operations: if two blocks are ordered in the POEG because of explicit coordination, their tags are unordered. Therefore, an additional mechanism is needed to record execution orderings imposed by coordination. To this end, a coordinatıon list is associated with each executing block $b$ and consists of the tags of the ancestors of $b$ such that all tags are unordered. The test for concurrency between two blocks $b$ and $c$ requires determining if $\operatorname{tag}_{b}$ or any of the tags in list $t_{b}$ are ordered with $\operatorname{lag}_{c}$. Thus, the total amount of work is bounded by the size of the coordination lists times the length of the tags.

The length of a coordination list is bounded by the maximum concurrency of the POEG. When a block terminated, its coordination list is deleted so that the total number of coordination lists is also bounded by the maximum concurrency of the POEG. If tags are stored directly in the access history, storage can potentially be large and there is a larger constant overhead because tags are variable length. On the other hand, if tags are stored indirectly (i.e. if access histories consist of pointers to tags), tags must be saved throughout the execution requiring storage proportional to the number of blocks.

Figure 4 illustrates the use of English-Hebrew labels for a POEG. The coordination list of block 1131,1231 contains the tag 121,113 because of the coordination edge in the POEG. Likewise, blocks 1211, 1131 and 123,113 have coordination lists containing the tag 113,123 . However, block 123,123 does not have a coordination list because all of the tags in the coordination lists of its parent blocks are ordered with 123,123. We can determine that blocks 111,123 and 12,11 are concurrent because their tags are unordered; namely, the first condition is satisfied: $111<12$ and $123>11$. However, blocks 1131,1231 and 12,11 are not concurrent because an entry in the concurrency list of 1131,1231 121,113 - is ordered with 12,11 .

\section{Task Recycling Algorithm}

Task Recycling reduces the cost of testing whether two blocks are concurrent to an array reference while increasing the expense of concurrency information maintenance. Instead of a tag, each block has a unique task identifier, which consists of a task and a version num. ber. Tasks can be recycled; that is, more than one block

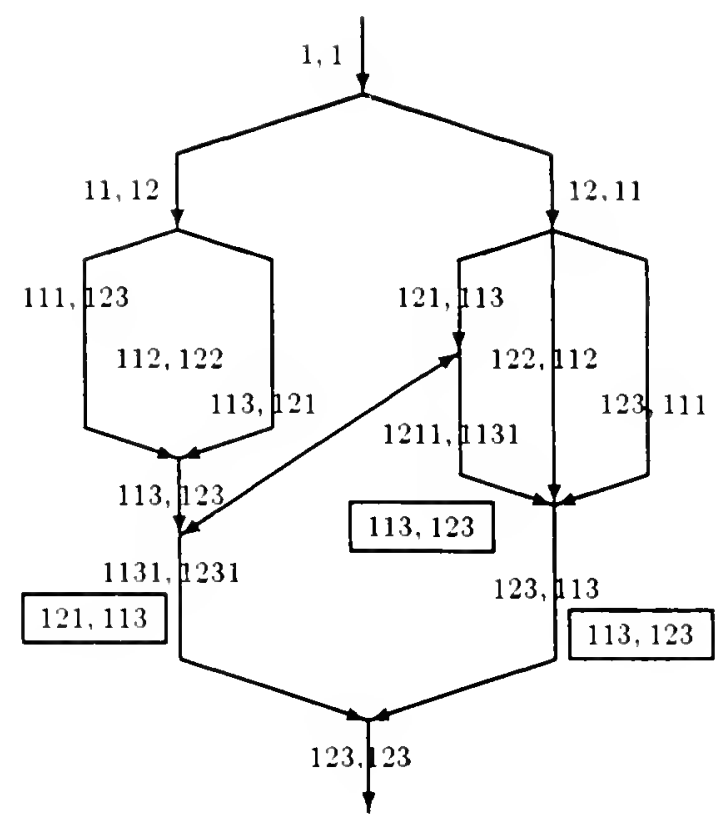

Figure 4: English-Hebrew Labeling

can be assigned to the same task at different times in the program execution. The version number of a task identifier is used to distinguish among different blocks assigned to the same task; every time a block is assigned to a task $t$, the associated version number $v$ is incremented. The label storage problem is therefore addressed because the number of labels is bounded and they are easy to store directly.

Concurrency information is maintained in a parent vector which is associated with each currently executing block $^{4}$. The number of entries in a parent vector is equal to the number of tasks. The $t^{\text {th }}$ entry in the parent vector for block $b$ contains the largest version number associated with those ancestors of $b$ which were assigned to task $t$. New parent vectors are formed from those vectors associated with parent blocks. In particular, when blocks $p_{1} \ldots p_{m}$ with task identifiers $t_{1} v_{1} \ldots t_{m} v_{m}$ create a new block $c$ the parent vector of $c$ is initialized as follows:

$$
\begin{aligned}
& \text { for } i=1 \text { to } T \text { do } \\
& \text { if } \left.\exists_{t, \in\left\{s_{1}\right.} s_{m}\right\}: i=t_{j} \text { then } \\
& \text { parent }[i] \leftarrow v_{j} \\
& \text { else } \text { parent }_{c}[i] \leftarrow \max \left(\text { parent }_{p_{1}}[i], \ldots \text { parent }_{p_{m}}[i]\right) \\
& \text { endfor }
\end{aligned}
$$

$A$ block $b$ is concurrent with a block with task identifier $t_{v}$ iff paren $t_{b}[t]<v$. Thus, there is a constant cost (an array access) for checking whether two blocks are con-

\footnotetext{
'Parent vectors correspond to before vectors in $[3,8]$. However. because we monitor on-line, we do not need the corresponding after vectors used for post-mortem trace analysis.
} 
current. (Coordination lists, on the other hand, must be searched linearly). As with English-Hebrew labels, only currently executing blocks need parent vectors; storing a task identifier in an access history is sufficient to determine whether two blocks are concurrent. Once a block terminates, its parent vector is discarded.

Figure 5 shows a task assignment and parent vectors for a POEG. In this example, block $1_{3}$ is concurrent

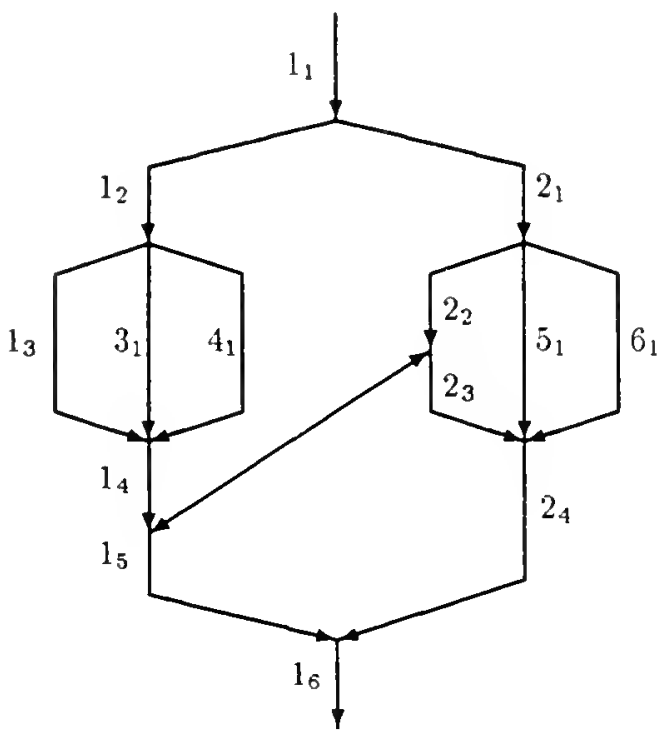

\begin{tabular}{||c|c||}
\hline Task Ids & Parent Vector \\
\hline $1_{1}$ & {$[0,0,0,0,0,0]$} \\
$1_{2}, 2_{1}$ & {$[1,0,0,0,0,0]$} \\
$1_{3}, 3_{1}, 4_{1}$ & {$[2,0,0,0,0,0]$} \\
$2_{2}, 5_{1}, 6_{1}$ & {$[1,1,0,0,0,0]$} \\
$1_{4}$ & {$[3,0,1,1,0,0]$} \\
$1_{5}, 2_{3}$ & {$[4,2,1,1,0,0]$} \\
$2_{4}$ & {$[4,3,1,1,1,1]$} \\
$1_{6}$ & {$[5,4,1,1,1,1]$} \\
\hline
\end{tabular}

Figure 5: Task Recycling Assignment

with block 2 , since the second entry in the parent vector of $1_{3}-[2,0,0,0,0,0]-$ is less than 1 . However, block $1_{5}$ is not concurrent with 2 , because the second entry in its parent vector - $[4,2,1,1,0,0]$ - is greater than 1 .

A block $b$ is validly assigned to a task $t$ iff it is not concurrent with any other block previously assigned to $t$. The goal of task assignment is to minimize the number of tasks used while maintaining a valid task assignment since this determines the length of parent vector. Our task assignment algorithm - from which this method derives its name - is based on a "most recently used" (MRU) heuristic and yields a good task assignment; there is no on-line task assignment algorithm which guarantees an optimal assignment [4].

To implement the MRU algorithm, a free task dag is maintained. There is a vertex in the dag associated with every doall, endall and coordination operation, and every currently executing block. (These latter vertices are always leaf vertices). The direction of edges in the dag is the reverse of the execution flow. A set of free tasks, i.e. those tasks that are available for recycling, is associated with each vertex. When a block $b$ terminates at a doall or endall operation $O$, it adds its task to the free task list of the vertex $v_{O}$ associated with $O$. When a block terninates in a coordination operation, its task is added to $v_{O}$ and the children its associated block $v_{b}$ are added to the child list of $v_{O}$. When a block $b$ is created after operation $O$, an edge is added from the vertex $v_{b}$ associated with $b$ to vertex $v_{O}$. To assign a task to a block $b$. a topological traversal of the dag is performed starting at $v_{b}$ until a vertex with a non-empty free task set is found.

Two optimizations minimize the cost of this topological traversal. Whenever a free task set of a vertex $v$ becomes empty, the dag is collapsed by deleting $v$ from the dag and adding edges from the parent vertices of $v$ to children vertices of $v$. Therefore, every vertex in the free task dag has at least one free task associated with it. Whenever a vertex $v$ has a single parent vertex $p, v$ is subsumed by $p$ by adding the free task set of $v$ to the free task set of $p$, deleting $v$ and adjusting the edges as appropriate.

Although the cost of performing task assignment is potentially quite high, for a large class of programs it is very limited. In particular, for programs with no nested parallelism and programs with nested parallelism but no coordination a constant amount of work is performed in assigning a task to a block.

\section{Empirical Results}

The preceding section presents two algorithms for detecting access anomalies in parallel programs. In general, English-Hebrew labeling will perform better than task recycling for programs with little coordination and frequent fine-grained doall operations because of the relatively high cost of maintaining parent vectors and task assignment. However, task recycling is clearly more efficient than English-Hebrew labeling in one way: the cost per variable access is less. Because this is frequently the most common operation, this is an important attribute of task recycling. Additionally, there is almost always a large storage penalty for saving tags in the English-Hebrew labeling algorithm. Moreover, if there is frequent coordination, the cost of maintaining coordination lists approaches the cost of maintaining parent vectors, so that any advantage of English-Hebrew labeling is diminished.

General discussions of algorithm design, however. give incomplete insight into the costs encountered when 
detecting anomalies in actual parallel programs. In order to better evaluate the two algorithins - as well as to gain insight into actual concurrency structure and memory access patterns - we monitored several benchmark scientific parallel programs:
Triso
- Solves a sparse triangular linear system of equations using wavefront parallelism
Finite - Solves a linear system using finite element methods
Simple - Solves partial differential equations for hydrodynamics and heat conduction
Polymer - Performs molecular dynamic calculations of polymer systems

Our first goal in performing these benchmarks is to obtain representative values for parameters that measure concurrency structure and shared variable access patterns for parallel program. This gives us a general idea about the applicability of various monitoring techniques. Our second goal is to measure the actual performance impact of monitoring parallel programs. EnglishHebrew and task recycling are compared on both their execution time and space requirements. The implementation and benchmark results are discussed in the following sections.

\subsection{Implementation}

The two monitoring algorithms are implemented for the Ultracomputer parallel Fortran compiler. The access anomaly detection algorithms are implemented by two library packages mostly written in $\mathrm{C}$. The routines for maintaining access histories are written in assembler for reasons of efficiency. A front-end pre-processor allocates the storage for access histories and inserts calls to the run-time libraries in the program being monitored. This implementation is relatively portable to other parallel Fortran system. The pre-processor approach also simplifies the implementation; however, the resulting monitoring efficiency is worse than if the compiler were modified.

Several optimizations were made to task recycling and English-Hebrew algorithms during implementation:

- For every monitored variable there is a "mirror" variable which contains its access history. Thus, when a block reads $A[i]$, the access history in $A_{a h}[i]$ is checked for anomalies and then updated. The access history lengths are fixed because of 16 megabyte memory constraints. While this limitation may result in undetected anomalies, we believe that anomalies will be missed only rarely, since the measurements discussed in Section 5.3 indicate that the size of the reader set is generally very smal! Each entry in an access history contains a task identifier or a pointer to the English-Hebrew label and the line number and a pointer to the function name of the last access; this enables us to identify the lo- cations in the program code where access a nomalies occur.

- English-Hebrew labels consist of a string of two byte integers and a length field. They are stored indirectly in access histories and coordination lists. This decreases the size and complexity of access histories. There is no attempt made to reclaim space for labels no longer referenced. We feel the additional expense of maintaining reference counts outweighed the benefits of possibly decreasing storage requirements.

- Blocks can share parent vectors (in task recycling) or coordination lists (in English-Hebrew labeling) if they have identical sets of ancestors. For example. all blocks created by a doall operation share the same ancestors; one of these blocks must obtain a private parent vector or coordination list only if it terminates before the endall (e.g. by performing a nested doall or coordination operation). For a nested doall operation with outer parallelism of $m$ and inner parallelism of $n$, this reduces the number of parent vectors and coordination lists by a factor of $n m{ }^{5}$

- Both algorithms benefit from a "run-untilcompleted" scheduling paradigm, which is the common scheduling method used in the Ultracomputer and many other parallel Fortran environments. Parallel scientific codes typically exhibit a high degree of nominal parallelism. In the run-untilcompleted model, each block created in a doall will not block until it terminates at its associated endall operation. This limits the number of blocks which can ever run concurrently to $n$, the underlying parallelism of the machine. This is not a strong optimization for English-Hebrew labeling, since the majority of its space requirement is for block labels: it is more effective for task recycling since parent vector storage is more significant.

- In the Ultracomputer parallel Fortran environment. each of $n$ actual processes perform the work of several parallel blocks. The differences among the parent vectors of these blocks are very small. Therefore, in order to reduce the work performed in maintaining parent vectors, a private "template" parent vector is cached for each level of nesting. When a block $b$ updates its parent vector, the process which is performing the work of $b$ also records the modifications. When block $b$ terminates it must update the parent vectors of its children blocks. Instead of comparing all of the entries in its parent vector. it simply compares the recorded modifications.

\footnotetext{
"If local memory is available and the cost of accessing shared memory is much higher than local memory, sharing of data structures may not be cost-effective.
} 

Likewise, when a new block is created the template parent vector must be reinitialized. The modification records are used to back out the changes made rather than reinitializing all of the entries.

For programs with only doall and endall operation, the per block cost is reduced from the maximum concurrency of the graph to the maximum level of nesting (the number of times that a change must be either added or backed out of a parent vector) and the amount of space and work per process is the product of the maximum concurrency and the level of nesting. Because coordination lists would have to be traversed to perform the updates, there is no corresponding optimizations for the EnglishHebrew labeling scheme.

\subsection{Concurrency Structure}

For all four benchmark parallel programs, the concurrency structure is quite simple: there is very limited nesting of doall constructs and minimal synchronization. However the degree and granularity of parallelism vary considerably.

- Triso has coarse granularity parallelism with limited synchronization. It consists of a single doall operation which creates 8 parallel threads; these subsequently perform two barrier synchronization operations.

- Simple has medium granularity parallelism with some locking. 1t performs 10 doall operations that each create 124 parallel threads, and 130 operations that create from 10 to 30 threads. In addition, during 10 phases of execution approximately half of the 30 concurrent threads obtain a lock (represented by an asynchronous coordination edge in the POEG).

- Finite exhibits a large degree of fine granularity parallelism and does not use coordination operation. It performs 60 doall operations that each create 1000 parallel threads; 50 operations that create 250 threads and 200 operations that create between 2 and 32 threads. Each block performs a very limited amount of computation; in many case a block consists of a single operation on an array element.

- Polymer also exhibits a large degree of fine granularity parallelism and does not use coordination operation; however, it has one level of nested doall operations (the first three benchmark programs have no nested doall operations). It performs 40 nested doall operations; the outer operations create 1000 parallel threads each of which creates 3 parallel subthreads. In addition, it performs 20 doall operations which create 350 parallel threads and 10 doall operations which create 100 parallel threads.

The last two programs have such fine granularity parallelism that monitoring them primarily measures the cost of maintaining concurrency information rather than the cost of checking accesses to shared variables. Table 1 summaries the concurrency parameters for the benchmark programs. The experimental results presented in

\begin{tabular}{||c||r|r|r||}
\hline Program & $\begin{array}{r}\text { Total \# } \\
\text { Blocks }\end{array}$ & $\begin{array}{r}\text { Max } \\
\text { Concur }\end{array}$ & $\begin{array}{r}\text { Average } \\
\text { Concur }\end{array}$ \\
\hline Triso & 24 & 8 & 8 \\
Simple & 4090 & 124 & 28 \\
Finite & 74,900 & 1,000 & 245 \\
Polymer & 128,000 & 3,000 & 1,800 \\
\hline
\end{tabular}

Table 1: Concurrency Structure

Sections 5.4 and 5.5 show that the concurrency structure of the program has a significant impact on the cost of maintaining concurrency information. However, the coneurrency structure does not significantly impact the cost per variable access, as discussed in the next section.

\subsection{Shared Variable Access Patterns}

The work and space required for maintaining access historles is proportional to the average size of the reader set of an access history. While theoretically the size of the reader set may grow to the maximum concurrency of the graph, in practice the size is much smaller. Many parallel scientific codes distribute workloads by partitioning data among concurrent threads. Hence a thread often shares data with a neighboring thread, but seldom shares data with all other threads. Therefore, one would expect the number of concurrent readers to be limited.

The shared memory access patterns measured for the benchmark programs support this conclusion and are shown in Table 2. Each column gives the percentage of accessed ${ }^{6}$ shared variables with at most the specified number of concurrent readers. In the Triso program. for example, $15 \%$ of the shared variables have two concurrent readers at some point during execution, but never have more than two.

The last column of Table 2 shows the average maximum reader set size for all variables with less than 10 concurrent readers ${ }^{\top}$. For the first three benchmark programs, this includes virtually all shared variables and is an upper bound on the size of the reader set. Since for the Polymer program $10 \%$ of the variables have more than 9 concurrent readers, the figure in the last column only reflects statistics for $90 \%$ of its shared variables If we are conservative and assume that all of these varl. ables are accessed by all 3,000 concurrent block, the average reader set size is 279 concurrent readers ( $w$ hich

\footnotetext{
${ }^{B}$ Because fixed maximum sized arrays are used. many shared variables are never accessed.

${ }^{7}$ It is not possible to measure reader sets larger than 10 due to memory constraints on the Ultracomputer
} 


$$
6
$$




\begin{tabular}{||c||r|r|r|r|r|r|r|r|r|r|r||r||}
\hline \multicolumn{1}{|c||}{} & \multicolumn{8}{|c||}{ Ave } \\
\cline { 2 - 11 } Program & 1 & 2 & 3 & 4 & 5 & 6 & 7 & 8 & 9 & $>9$ & Size \\
\hline Triso & $80.2 \%$ & $15.0 \%$ & $4.8 \%$ & $0.0 \%$ & $0.0 \%$ & $0.0 \%$ & $0.0 \%$ & $0.0 \%$ & $0.0 \%$ & $0.0 \%$ & 1.25 \\
Simple & $49.3 \%$ & $41.0 \%$ & $9.6 \%$ & $\dagger$ & $0.0 \%$ & $0.0 \%$ & $0.0 \%$ & $0.0 \%$ & $0.0 \%$ & $\dagger$ & 1.69 \\
Finite & $48.4 \%$ & $32.5 \%$ & $4.6 \%$ & $\dagger$ & $\dagger$ & $0.9 \%$ & $\dagger$ & $\dagger$ & $13.4 \%$ & $\dagger$ & 2.71 \\
Polymer & $72.9 \%$ & $9.4 \%$ & $2.6 \%$ & $1.1 \%$ & $2.8 \%$ & $0.8 \%$ & $2.6 \%$ & $6.2 \%$ & $2.0 \%$ & $9.3 \%$ & 1.31 \\
\hline
\end{tabular}

Table 2: Reader Set Sizes

is still much less than 3,000 ). If we are slightly less conservative and assume that all of these variables are accessed by the average number of concurrent blocks, the average reader set size is 168 .

Table 2 reveals two somewhat surprising results:

1. The size of reader sets tends to be very small. In all four programs, more than $80 \%$ of the variables are never read by more than two concurrent readers and almost $50 \%$ are never read concurrently. Moreover, a variable with maximum measured reader set size of $n$ may actually have a much smaller reader set size throughout most of the execution of the program. Hence our estimate of the average reader set size is pessimistic with respect to the actual average reader set size.

2. There appears to be little correlation between the average reader set size and the degree of parallelism of the program. For example, the Triso and Polymer programs have fairly similar access patterns for the majority of their variables (excepting the $9.3 \%$ in the Polymer program with more than 9 concurrent readers). Triso, however, has a very low degree of parallelism while Polymer has nested parallelism of a very high degree.

Since the average reader set size is so much smaller than their worst case bounds, we conclude that the access history based algorithms are preferable to that of [11] in which the storage depends on the maximum concurrency of the POEG.

\subsection{Space Requirements}

For the results described in the following two sections four versions of each program were executed:
Unmonator
- unmonitored program
Monitor(1)
- monitors every shared variable using a reader set size of one
Monator(2)
- monitors every shared variable using a reader set size of two
Concurrency - maintains concurrency information only; no variables are monitored

Table 3 compares the memory requirements for each of the four versions of the benchmark programs listed above. The static size is the size of the object mod-

\begin{tabular}{||c||r|r|r|r|r||}
\hline \multicolumn{1}{||c||}{ Program } & \multicolumn{3}{c|}{ Statıc Sizes } & \multicolumn{2}{c||}{ Dynamıc } \\
\cline { 2 - 6 } & Unm & Mon(1) & Mon(2) & \multicolumn{1}{c|}{ TR } & E-H \\
\hline Triso & 163 & 660 & 1,000 & 2 & 8 \\
Simple & 314 & 921 & 1,270 & 6 & 80 \\
Finite & 230 & 745 & 1,020 & 29 & 954 \\
Polymer & 639 & 2,898 & 4,461 & 279 & $7.000 \dagger$ \\
\hline
\end{tabular}

Table 3: Space Requirements (in Kbytes)

ule which includes additional monitoring code as well as storage needed for access histories. (This is virtually the same for both algorithms). The dynamic size is the amount of storage needed for concurrency information, which is allocated at run-time. As is seen in Table 3, English-Hebrew labeling requires substantially more space than task recycling for maintaining concurrency information. In fact, it is not possible to obtain the actual dynamic memory requirements of Polymer for the English-Hebrew labeling scheme; the amount of storage needed for concurrency information exceeds the capacity of the Ultracomputer and hence an estimate was calculated based on the concurrency structure of the POEG.

\subsection{Execution Times}

Table 4 displays user mode execution times for the four benchmark programs using both of the anomaly detec. tion algorithms. Concurrency isolates the cost of main-

\begin{tabular}{|c|c|c|c|c|}
\hline \multicolumn{5}{|c|}{ Task Recycling } \\
\hline Program & Unm & Conc & .Mon(1) & .Hon(2) \\
\hline Triso & 3.5 & 4.1 & 19.6 & 227 \\
\hline Simple & 202 & 263 & 550 & 598 \\
\hline Finite & 98 & 734 & 959 & 1108 \\
\hline Polymer & 789 & 3330 & 4367 & 4607 \\
\hline \multicolumn{5}{|c|}{ Enghsh-Hebrew $\dagger$} \\
\hline Program & $U n m$ & Conc & $\operatorname{Mon}(1)$ & .Hon(2) \\
\hline Triso & 3.5 & 4.7 & 20.9 & 244 \\
\hline Simple & 202 & 237 & 579 & 676 \\
\hline Finite & 98 & 407 & 704 & 1072 \\
\hline
\end{tabular}

polymer was not monitored due to memory constrants

Table 4: Total Execution Time (in seconds) 
taining the concurrency information from the overall cost of detecting access anomalies. This cost is substantial for the programs with very high degrees of finegrained parallelism. In the current implementation of the task assignment algorithm, assigning and freeing a task requires locking vertices in the free task dag, which creates a serial bottleneck. If free tasks are stored in parallel access data structures (e.g. parallel access queues), it may be possible to decrease this serialization effect.

For the Triso and Simple programs, the EnglishHebrew labeling algorithm requires more time than task recycling for monitoring variables (as is shown in the run times for Monztor(1) and Monztor(2)), even when the cost of maintaining concurrency information is less (as in shown in the run times for Concurrency). The primary cost in the Polymer and Finite programs is the maintenance of concurrency information; this is not surprising given the fine granularity of the parallelism in the two programs. For both algorithms, the overall increase in computition time is too high for transparent monitoring of all shared references. Nevertheless, we not believe the cost it too prohibitive for on-the-fly anomaly detection to be a useful debugging tool. Moreover, static analysis and/or a compiler-based implementation should make it more efficient.

Note that the time measurements presented are the total execution times of all concurrent threads rather than elapsed running time. The maintenance of concurrency information results in a $57 \%-160 \%$ increase in elapsed running time, while monitoring all accesses to all shared variables with reader sets of size one incurs a $160 \%$ to $460 \%$ increase in execution times. The difference between the elapsed and execution time increases stems from Amdahl's law: almost all of the additional work is performed in parallel.

From Section 5.3 we know that using two entry reader sets instead of one entry reader sets significantly increases the percentage of variables guaranteed to have at least one anomaly detected (from $50 \%$ to $80 \%$ ). A comparison of the execution times for Monutor(1) and Monitor(2) in Table 4 shows that we may do so with little additional cost. Table 5 isolates the cost of maintaining access histories and shows the percentage increase when using reader sets of size two instead of one. The isolated times are computed as the total monitored

\begin{tabular}{||c||c|c|c|c|c|c||}
\hline \multirow{2}{*}{\multicolumn{1}{|c||}{ Program }} & \multicolumn{2}{c|}{ Mon(1) } & \multicolumn{2}{c|}{ Mon(2) } & \multicolumn{2}{c||}{ Increase } \\
\cline { 2 - 7 } & $T R$ & $E-H$ & $T R$ & $E-H$ & $T R$ & $E-H$ \\
\hline Triso & 16.4 & 16.3 & 21.0 & 22.1 & $20 \%$ & $22 \%$ \\
Simple & 287 & 242 & 335 & 339 & $17 \%$ & $40 \%$ \\
Finite & 225 & 297 & 354 & 685 & $57 \%$ & $130 \%$ \\
\hline
\end{tabular}

Table 5: Access History Update Time (in seconds)

execution time (Monator(1) and Monator(2)) less the ex- ecution time when simply maintaining concurrency information (Concurrency). These relative time increases indicate that as reader sets grow in size, the cost of detecting anomalies increases more rapidly for EnglishHebrew labeling than for task recycling. Therefore, as larger reader sets are used the cost of the more complex task concurrency verification in the English-Hebrew algorithm becomes increasingly more significant.

\section{Concluding Remarks}

This paper presents the problem of detecting access anomalies in parallel programs under a models of concurrency which incorporates nested parallel loops and synchronous and asynchronous coordination. An existing on-the-fly algorithm - English-Hebrew labeling - is described and compared with a new algorithm based on task assignment to a partial order execution graph. Experimental data from monitoring four benchmark scientific programs using the two algorithms indicate four important results:

1. The benchmark programs use data partitioning so extensively that over $80 \%$ of all variables never have more than two concurrent readers. Therefore, the size of access histories appears to be independent of the degree of parallelism within the program.

2. The task recycling algorithm requires much less storage for concurrency information than EnglishHebrew labeling.

3. Because of its efficient concurrency information management, English-Hebrew labeling performs better on programs with frequent doall operations and relatively few accesses to shared variable.

4. However, as reader sets or concurrency lists increase in size the high cost of performing concurrency verification in English-Hebrew labeling outweighs the cost of parent vector management in the task recycling algorithm.

If the benchmark programs are indicative of a wide class of parallel programs, the task recycling algorithm is an important improvement over existing technology.

\section{Acknowledgements}

We would like to thank Marc Snir and Larry Rudolph for their introduction to this problem and Ron Cytron, Allan Gottlieb, Bud Mishra and Ed Schonberg for their many helpful suggestions.

\section{References}

[1] Todd R. Allen and David A. Padua. Debugging Fortran on a Shared Memory Machine. In Proceedings of the International Conference on Parallel Processing, pages $i 21-i 1 T$, Aug 198 $i$. 

[2] David Callahan and Jaspai Subhlok. Static Analysis of Low Level Synchronization. In Proceedings on the SIGPLAN Workshop on Parallel and Distributed Debugging, pages 100-111, May 1988.

[3] Jong-Deok Choi, Barton P. Miller, and Robert Netzer. Techniques for Debugging Parallel Programs with Flowback Analysis. Technical Report, University of Wisconson, Aug 1988.

[4] Anne Dinning and Edith Schonberg. An Evaluation of Monitoring Algorithms for Access Anomaly Detection. Technical Report Ultracomputer Note \#163, New York University, July I989.

[5] Perry A. Emrath and David A. Padua. Automatic Detection of Nondeterminancy in Parallel Programs. In Proceedings on the SIGPLAN Workshop on Parallel and Distributed Debugging, pages 8999, May 1988.

[6] Allan Gottlieb. An Overview of the NYU Ultracomputer Project. In J.J. Dongarra, editor, Experimental Parallel Computing Architectures, pages 25 - 95, Elsevier, 1988.

[7] Leslie Lamport. Time, clocks, and the ordering of events in a distributed system. Communications of the ACM, 21(7), Jul 1978.

[8] Barton P. Miller and Jong-Deok Choi. A Mechanism for Efficient Debugging of Parallel Programs. In Proceedings on the SIGPLAN Workshop on Parallel and Distributed Debugging, May 1988.

[9] Itzhak Nudler and Larry Rudolph. Indeterminancy Considered Harmful. 1988.

[10] Itzhak Nudler and Larry Rudolph. Tools for the Efficient Development of Efficient. Parallel Programs. In $1^{\text {st }}$ Israeli Conference on Computer System Engineering, 1988.

[11] Edith Schonberg. On-The-Fly Detection of Access Anomalies. In Proceedings on the SIGPLAN Con. ference on Programming Language Design and Implementation, Jun 1989.

[12] Marc Snir. Private correspondence. 1988. 

Variations of this result date back at least to 1966 and proofs are given in a number of papers; see e.g. Bramble [12], Bramble, Pasciak and Schatz [15] or Yserentant [58].

Since all the elements of $V_{i j}^{h}$ vanish at the vertices of the substructures, we must choose the interpolant $I_{H} u_{h}$ as the element in $V^{H}$ when, as required by Lemma 1 , we define the representation of $u_{h}$. It is easy to show that $\left|I_{H} u_{h}\right|_{H^{1}\left(\Omega_{i}\right)}^{2}$ can be estimated from above by

$$
\sum_{k, l \in \mathcal{V}_{1}}\left(u_{h}\left(x_{k}\right)-u_{h}\left(x_{l}\right)\right)^{2}
$$

where the $\mathcal{V}_{i}$ is the set of vertices of the substructure $\Omega_{i}$. It then follows from Lemma 2 that

$$
\left|I_{H} u_{h}\right|_{H^{1}\left(\Omega_{i}\right)}^{2} \leq \text { const. }(1+\log (H / h))\left|u_{h}\right|_{H^{1}\left(\Omega_{i}\right)}^{2} .
$$

For a bound on the other terms in the decomposition, required when using Lemma 1 , see Dryja and Widlund [28].

Using the method with local preconditioners developed in the end of Section 3, a number of algorithms can be derived from the basic method that we have just introduced. All that is needed is to replace the Dirichlet problem on $\Omega_{i j}$ by a preconditioner with a symmetric, positive definite coefficient matrix. A proof of the main result of Bramble, Pasciak and Schatz [15] can be obtained in this way by using (14), (15), Theorem 2 and a bound for the condition number of the two subregion problem defined on $\Omega_{i j}$. Such local bounds are given e.g. in Bjørstad and Widlund [9], Bramble, Pasciak and Schatz [18] and Dryja [22], [23].

In our previous paper, only equation (2) was considered. By using the formulas $(7),(8)$, and (9), the proof can be extended immediately to equation (3); the whole machinery can also be used locally. In this variant of the proof, only four subspaces play a role, namely, the three-dimensional space of linear functions on $\Omega_{i}$, and the restriction to $\Omega_{i}$ of the spaces $V_{i j}^{h}$ which correspond to the three edges of that substructure.

In preparation for the three-dimensional case, we develop an alternative basic algorithm for the two-dimensional problems. This algorithm is defined in terms of subspaces related to individual substructures. For any substructure $\Omega_{i}$, we keep the three local subspaces obtained as above from the spaces $V_{i j}^{h}$. For an interior substructure, we replace the three-dimensional space of linear functions, used previously, by the three-dimensional space spanned by

$$
\psi_{k}^{(i)}=\varphi_{k}-\beta_{k}^{(i)} \sum_{l \in \mathcal{V}_{l}} \varphi_{l}+\beta_{k}^{(i)}, k \in \mathcal{V}_{i}
$$

Here $\varphi_{k}$ is the standard nodal basis function associated with an element of $\mathcal{V}_{i}$. We note that, just as the regular nodal basis functions, $\psi_{k}^{(i)}\left(x_{l}\right)=\delta_{k l}, \quad k, l \in \mathcal{V}_{i}$. The positive constants $\beta_{k}^{(i)}$ are chosen below, so that

$$
\sum_{k \in \mathcal{V}_{\mathrm{r}}} \beta_{k}^{(i)}=1
$$

Then,

$$
\sum_{k \in \mathcal{V}_{1}} \psi_{k}^{(i)}=1
$$


This subspace, thus, also contains the constants, i.e. the null space of the elliptic operator restricted to $\Omega_{i}$ with a Neumann boundary condition. (When these methods are extended to elasticity problems, we similarly have to include the whole null space of that operator in the space that accounts for the global transportation of information.) For a substructure that intersects the boundary, we simply use the space spanned by the standard basis functions $\varphi_{k}$ corresponding to the vertices of the substructures where a Dirichlet condition is not imposed. For the correct definition of boundary substructures for the case of three dimensions; see below.

An elementary computation shows that, for an interior substructure, the quadratic form associated with this subspace is given by

$$
\sum_{k \in \mathcal{V}_{1}} k_{k k}^{(i)}\left(u_{h}\left(x_{k}\right)-\bar{u}_{h}^{(i)}\right)^{2}
$$

Here, $\bar{u}_{h}^{(i)}$ is the weighted average of $u_{h}\left(x_{k}\right), k \in \mathcal{V}_{i}$, with the weights $\beta_{k}^{(i)}$. It is easy to see that this quadratic form can be bounded from above and below by the form (17) given in the discussion of the previous algorithm.

Inspired by Mandel [36], we now introduce the quadratic form

$$
\mathcal{J}\left(u_{h}, y\right)=\sum_{i} \sum_{k \in \mathcal{V}_{1}} k_{k k}^{(i)}\left(u_{h}\left(x_{k}\right)-y^{(i)}\right)^{2}-2 \sum_{i} \sum_{k \in \mathcal{V}_{1}} u_{h}\left(x_{k}\right) b_{k}^{(i)} .
$$

For substructures which have at least one vertex on $\partial \Omega$, we set $y^{(i)}=0$. In three dimensions, we adopt the same rule for all substructures which have at least an edge on $\partial \Omega$, but treat those which only touch the boundary at individual points as if they were interior.

The vector $b^{(i)}$ corresponds to the part of the load vector which is associated with $\Omega_{i}$. Its components are computed by evaluating an appropriate functional at $\psi_{k}^{(i)}$.

It is easy to show that if

$$
\beta_{k}^{(i)}=\frac{k_{k k}^{(i)}}{\sum_{l \in \mathcal{V}_{1}} k_{l l}^{(i)}}
$$

then,

$$
\bar{u}_{h}^{(i)}=\arg \min _{y^{(1)}} \sum_{k \in \mathcal{V}_{1}} k_{k k}^{(i)}\left(u_{h}\left(x_{k}\right)-y^{(i)}\right)^{2} .
$$

Therefore, the solution of the linear system corresponding to these subspaces can be obtained by minimizing $\mathcal{J}$ with respect to $u_{h}$ and $y$. By setting the gradient of $\mathcal{J}$ equal to zero, we obtain a linear system of equations, which naturally can be written in a two-by-two block form. The diagonal blocks are diagonal with elements $\sum_{i} k_{k k}^{(i)}$ and $\sum_{k \in \mathcal{V}_{1}} k_{k k}^{(i)}$, respectively. The off-diagonal blocks are sparse and are also given by $k_{k k}^{(i)}$.

Since the diagonal blocks are diagonal, it is easy to eliminate all the variables $u_{h}\left(x_{k}\right)$. This elimination step results in a sparse system, similar to that of a finite difference problem on a coarse mesh that is dual to the one used to define the space $V^{H}$. It is often feasible and economical to solve this system by a direct method. Once the values of $\bar{u}_{h}^{(i)}$ are known, it is easy to determine the values of $u_{h}\left(x_{k}\right), k \in \mathcal{V}_{i}$, and the 
corresponding element of the subspace. The contributions of the other subspaces can now be computed, while observing that we must make the sum of all the contributions continuous across the interfaces between the substructures.

Remark. The method just outlined draws heavily on the work of Bramble, Pasciak and Schatz [16]. However, the current algorithm uses a different average and basis. Our theory does not require that the linear system, which determines the averages, to have a $M$-matrix. Our method can therefore be extended to the elliptic systems of elasticity; cf. also Mandel [36].

It can be shown, straightforwardly, that there is no longer a need to insist on triangular substructures. In the general case, we can simply redefine $\mathcal{V}_{i}$ as the set of nodal points which belong to the boundaries of at least three substructures.

Lemma 2 holds only in two dimensions; it resembles a Sobolev inequality which is far from valid in three dimensions. However, for finite element functions, we can find a useful bound of the $L_{2}$ - norm over an interval in terms of the strain energy. Let $\mathcal{W}_{i}$ denote the wire basket of the substructure $\Omega_{i}$. This is the union of the edges of the tetrahedral substructure; more generally, it is defined as the set of nodal points of $\Omega_{i}$, which belong to the boundaries of at least three substructures. The following lemma is essentially a corollary of Lemma 2 .

LEMMA 3. Let $\alpha$ be any convex combination of values of $u_{h}(x), x \in \mathcal{W}_{i}$. Then

$$
h\left\|u_{h}-\alpha\right\|_{l^{2}\left(\mathcal{W}_{1}\right)}^{2} \leq \text { const. }(1+\log (H / h))\left|u_{h}\right|_{H^{1}\left(\Omega_{i}\right)}^{2} .
$$

An algorithm for the three-dimensional case can now be defined in terms of the subspaces $V_{i j}^{h}$, which are defined just as in the two-dimensional case, and a special subspace. This subspace is given in terms of a basis defined as in (19), where we replace the set $\mathcal{V}_{i}$ by $\mathcal{W}_{i}$. The study of the linear algebra problem associated with this basis proceeds very much as in the two-dimensional case. We only note that when we compute the expression corresponding to (20), we also get off-diagonal terms corresponding to nodes on $\mathcal{W}_{i}$, which are next neighbors. It is however easy to show that these contributions can be disregarded. The rest of the analysis and the development now follows just as in the two-dimensional case. We note that the number of elements in $\mathcal{W}_{i}$ grows linearly with $H / h$. The nontrivial part of the sparse linear system of the special subspace has a dimension, which is equal to the number of interior substructures.

We have obtained the following result.

THEOREM 3. The operator $P$ of the additive algorithm defined by the spaces $V_{i j}^{h}$ and that defined by the basis functions given by (19), or the corresponding formula for the three-dimensional case, satisfies the estimate $\kappa(P) \leq$ const. $(1+\log (H / h))^{2}$ for equations (2) and (3). The constant is independent of $h, H$ and the discontinuities of the coefficient $\rho(x)$.

6. Schwarz Methods for Problems on the Interfaces. We have already seen that the original linear systems of equations can be reduced to smaller systems, where the remaining variables are associated with the boundaries $\partial \Omega_{i}$ of the substructures. We have also shown that this reduced set of variables are associated with the piecewise discrete harmonic functions. This is quite similar to potential theory for elliptic problems where there is also a reduction in dimension. 
Here, we only consider the first model case (2), since at this time, we do not know if bounds independent of the variations of the coefficients of equation (3) can be obtained for the methods that we are going to introduce. We denote by $V_{\text {harm }}^{h}(\Gamma)$ the space of piecewise discrete harmonic functions defined by the values of the finite element functions on the set

$$
\Gamma=\bigcup \partial \Omega_{i} \backslash \partial \Omega
$$

The space of traces $H^{1 / 2}(\Gamma)$ are the restrictions of $H^{1}(\Omega)$ to $\Gamma$. As is well known, the norm of this space can be defined by

$$
|u|_{H^{1 / 2}(\Gamma)}^{2}=\sum_{i}|u|_{H^{1 / 2}\left(\partial \Omega_{1}\right)}^{2}
$$

where

$$
|u|_{H^{1 / 2}\left(\partial \Omega_{1}\right)}^{2}=\int_{\partial \Omega_{1}} \int_{\partial \Omega_{1}}\left(|u(x(s))-u(x(t))|^{2} /|x(s)-x(t)|^{d}\right) d s d t .
$$

Here, $d=2,3$, for problems with $\Omega \subset R^{d}$.

We consider several different additive Schwarz methods. The first is defined by the partitioning

$$
V_{h a r m}^{h}(\Gamma)=\sum_{i} V_{h a r m, i}^{h}
$$

where $V_{h a r m, i}^{h}$ is the subspace of $V_{h a r m}^{h}(\Gamma)$ with zero values on $\Gamma$ outside $\partial \Omega_{i}$. By using the same technique as in the previous three sections, it is easy to see that the operator $P$. which correspond to this set of subspaces, is bounded uniformly from above. We can use the techniques of Widlund [54] and Dryja [24] for the two- and three-dimensional cases, respectively and Lemma 1 , to obtain the bound $C_{0}^{2} \leq$ const. $(1+\log (H / h))^{2}$. It is not possible to obtain a uniform bound, since then the spaces $H_{00}^{1 / 2}$ and $H^{1 / 2}$ would have to be the same; cf. Lions and Magenes [33].

The computations associated with the space $V_{h a r m, i}^{h}$ involve piecewise discrete harmonic functions, which differ from zero in all the substructures which are next neighbors of $\Omega_{i}$. The coefficient matrix of the linear system, which is solved when computing the projection of $V_{\text {harm }}^{h}$ onto $V_{\text {harm,i }}^{h}$ is the principal minor of $S$ associated with the nodes on $\partial \Omega_{i}$. It also involves contributions from the matrices $S^{(j)}$ of the neighbors of $\Omega_{i}$. It is natural to replace this matrix by $S^{(i)}$, since the use of this preconditioner only involves a problem on one substructure.

What we have just given is an alternative derivation of an algorithm introduced by Bourgat, Glowinski, Le Tallec and Vidrascu [11]. We note that there is a minor technical issue related to the fact that $S^{(i)}$ is singular for interior substructures, but that it is easy to solve that problem. Using the formalism developed in Section 2, we can write down the following formula for the preconditioner which corresponds to this algorithm,

$$
\left.x_{B}^{T} \hat{S}^{-1} y_{B}=\sum x_{B}^{(i)^{T}} S^{(i)}\right)^{-1} y_{B}^{(i)} .
$$

Using Lemma 3.2 in Widlund [54], the corresponding estimates developed for the three-dimensional case in Dryja [24] and the estimate (16), we can conclude the proof of the following result. 
THEOREM 4. The operator $\hat{P}$ of the additive algorithm defined by the spaces $V_{\text {harm,i, }}$ applied to equation (2), and the local preconditioner defined by $S^{(i)}{ }^{-1}$ satisfies the estimate $\kappa(P) \leq$ const. $(1+\log (H / h))^{4}$. The constant in the estimate is independent of $h$ but not of $H$.

By using an elementary argument given in Widlund [54], it is easy to see that $\kappa(P)$ must grow at least as fast as $1 / H^{2}$, since in each iteration, information is exchanged only with neighboring substructures. The performance of the algorithm, for the case of many substructures, can be improved by introducing the same spaces of modest dimension, which provide some global transportation of information in the methods considered in Section 5. After this modification, the constant in the theorem can be shown to be independent of $H$ as well.

We note that in each iteration of this algorithm, both a Neumann and a Dirichlet problem have to be solved on each substructure. This results in twice as much work per iteration as for many other domain decomposition methods.

If there is a red-black ordering of the substructures, there is another way of analyzing this algorithm. We first consider the two subdomain case. We note that the inverse of the preconditioner corresponding to the Neumann-Dirichlet algorithm is $S^{(1)^{-1}}$ or $S^{(2)^{-1}}$ depending on the roles assigned to the substructures; cf. Bjørstad and Widlund [9]. We can therefore view the preconditioner introduced in this section as an average of Neumann-Dirichlet operators. In this simple case, the condition number remains uniformly bounded since there are no so-called cross points. Similarly, we can use a result by Dryja [24] to prove that the algorithm for three dimensions, which incorporates the global subspace introduced in the previous section, has a condition number which is bounded by const. $(1+\log (H / h))^{3}$. This again follows from the observation that this new preconditioner can be viewed as an average of two instances of the operator which arises in the study of the version of the Neumann-Dirichlet algorithm considered in Dryja [24].

We conclude this section by a brief discussion of some recent work by Barry Smith, a Courant Institute graduate student. His algorithm, described in Smith [48], is an additive Schwarz method, which uses the subspace $V^{H}$ both in two and three dimensions. We give some details only for the three-dimensional case. In addition to $V^{H}$, there are subspaces associated with each face, edge and vertex of the substructures. The subspace associated with a face is $H_{00}^{1 / 2}\left(\Gamma_{i j}\right) \cap V_{h a r m}^{h}$ and therefore closely related to the space $V_{i j}^{h}$ introduced in Section 5. Similarly, for an edge (vertex), Smith uses a space associated with a neighborhood in $\Gamma$ of the edge (vertex) which extends a distance on the order of the diameter of $\Omega_{i}$. The success of this approach can be explained informally by noting that the overlap is relatively generous. The numerical results, so far confined to two dimensions and simple substructures with benign aspect ratios, show great promise. In addition to Poisson's equation, membrane and shell models have been tested. So far, the condition numbers have always been less than 4 .

The following theoretical result has been established for both two and three dimensions. It also holds for elliptic systems such as those of linear elasticity; cf. Smith [48].

THEOREM 5. The operator $P$ of the additive algorithm developed by Smith satisfies the estimate $\kappa(P) \leq$ const. The constant in the estimate is independent of $h$ as well as $H$. 
7. Optimal Iterative Refinement Methods. In our study of iterative refinement methods, we consider problems on a special kind of composite finite element triangulations. We begin by introducing a relatively coarse triangulation of $\Omega^{(1)}=\Omega$, and denote the corresponding space of finite element functions by $V^{h_{1}}$. We can think of this space as having a uniform (or relatively uniform) mesh size $h_{1}$. Let $\Omega^{(2)}$ be a subregion where we wish to increase the resolution. We do so by subdividing the elements and introducing an additional finite element space $V^{h_{2}}$. This space is constructed quite similarly to the previous one and it contains $V^{h_{1}} \cap H_{0}^{1}\left(\Omega_{2}\right)$ as a subspace. We assure that the resulting composite space $V^{h_{1}}+V^{h_{2}}$ is conforming by having the functions of $V^{h_{2}}$ vanish on $\partial \Omega^{(2)}$. We repeat this process by selecting a subregion $\Omega^{(3)}$ of $\Omega^{(2)}$ and introducing a further refinement of the mesh and the finite element space, etc. We denote the resulting nested subregions and subspaces by $\Omega^{(i)}$ and $V^{h_{s}}$, respectively. Throughout, we assume that

$$
\Omega^{(i)} \subset \Omega^{(i-1)}
$$

and that

$$
V^{h_{1-1}} \cap H_{0}^{1}\left(\Omega^{(i)}\right) \subset V^{h_{1}} \subset H_{0}^{1}\left(\Omega^{(i)}\right), \quad i=2, \ldots, k .
$$

The composite finite element space, on the repeatedly refined mesh, is

$$
V^{h}=V^{h_{1}}+V^{h_{2}}+\ldots+V^{h_{k}} .
$$

The finite element models on composite meshes is thus systematically constructed by introducing a basic finite element approximation on the entire region and then selecting subregions, and subregions of subregions etc., where the finite element model is further refined in order to gain higher accuracy. In each iteration of the iterative refinement methods, problems representing finite element models on the original region and the subregions, prior to further refinement, are solved. As always, an additive algorithm is defined by specifying the subspaces $V_{i}$, or alternatively, the projections $P_{i}$.

In order to prove the results given at the end of this section, we need some additional technical assumptions. To make our proofs work, we cannot allow the sets $\Omega_{t-1} \backslash \Omega_{i}$ to become arbitrarily thin in comparison with the diameter of $\Omega_{i-1}$. We also assume that the area of any triangle on level $i$ can be bounded by const. $q^{i-j}$ times the area of the triangle on level $j$ of which it is a part. Here $q$ is a constant $<1$ and $j<i$.

The fundamental building blocks of our algorithms are the projections $P_{i}^{j}, j=$ $i-1, i$, onto the spaces $V^{h}, \cap H_{0}^{1}\left(\Omega_{i}\right)$. We note that if $j=i-1$, we solve a problem on $\Omega_{i}$ with a coarser mesh than if $V^{h_{1}}$ were used. The projection $P_{i}^{j}$ is defined in terms of the unique element of $V^{h}, \cap H_{0}^{1}\left(\Omega_{i}\right)$, which satisfies

$$
a\left(P_{i}^{\jmath} v_{h}, \phi_{h}\right)=a\left(v_{h}, \phi_{h}\right), \forall \phi_{h} \in V^{h} \cap \cap H_{0}^{1}\left(\Omega_{i}\right) .
$$

We consider two different algorithms and distinguish between them by using superscripts. The perhaps most natural algorithm uses the projections $P_{i}^{(1)}=P_{i}^{i}$. The condition number of this algorithm can grow as fast as linearly with $k$. By the standard argument, it is easy to show that the eigenvalues of $P^{(1)}$ are bounded from above by $k$. This bound is attained if $V^{h_{1}} \cap H_{0}^{1}\left(\Omega_{k}\right)$ is not empty, i.e. when the mesh size $h_{1}$ 
is fine enough. Any function in this space belongs to $V^{h_{\mathrm{i}}}, i=1,2, \ldots, k$, and is exactly reproduced by each of the projection operators. It is therefore an eigenfunction of $P^{(1)}$ with the eigenvalue $k$. Similarly, any function which belongs to $V^{h_{1}} \cap H_{0}^{1}\left(\Omega_{1} \backslash \Omega_{2}\right)$ is an eigenfunction with eigenvalue 1 . We have shown in [56], that the eigenvalues of $P^{(1)}$ are bounded from below by a constant. The condition number of $P^{(1)}$ is therefore of order $k$.

We are principally interested in the additive algorithm defined by the projections $P_{i}^{(2)}=P_{i}^{i}-P_{i+1}^{i}, i \leq k-1$, and $P_{k}^{(2)}=P_{k}^{k}$. It easy to show that these operators are projections and that the composite finite element space $V^{h}$ is the direct sum of the corresponding subspaces $V_{i}^{(2)}$. The following results have recently been demonstrated in Dryja and Widlund [27] and Widlund [56]. The latter paper also contains a similar result for a multiplicative algorithm.

THEOREM 6 . The condition number of $P^{(2)}$ is uniformly bounded by a constant. The condition number of $P^{(1)}$ grows at most linearly with the number of refinement levels.

We note, that Tarek Mathew [40] has developed iterative refinement methods for Raviart-Thomas finite element methods and obtained bounds for their rate of convergence.

\section{REFERENCES}

[1] V. I. Agoshkov. Poincaré-Steklov operators and domain decomposition methods in finite dimensional spaces. In Roland Glowinski, Gene H. Golub, Gérard A. Meurant, and Jacques Périaux, editors, First International Symposium on Domain Decomposition Methods for Partial Differential Equations, SIAM, Philadelphia, 1988.

[2] Ivo Babuska, Alan Craig, Jan Mandel, and Juhani Pitkäranta. Efficient Preconditioning for the p-Version Finite Element Method in Two Dimensions. Technical Report 41098, University of Colorado at Denver, October 1989. Submitted to SIAM J. Numer. Anal.

[3] Randolph E. Bank, Todd F. Dupont, and Harry Yserentant. The hierarchical basis multigrid method. Numer. Math., 52:427 - 458, 1988.

[4] K. Bell, B. Hatlestad, O. E. Hansteen, and Per O. Araldsen. NORSAM, a programming system for the finite element method. Users manual, Part 1, General description. NTH, Trondheim, 1973.

[5] Petter E. Bjørstad. Multiplicative and Additive Schwarz Methods: Convergence in the 2 domain case. In Tony Chan, Roland Glowinski, Jacques Périaux, and Olof Widlund, editors, Domain Decomposition Methods, SIAM, Philadelphia, 1989.

[6] Petter E. Bjørstad and Anders Hvidsten. Iterative methods for substructured elasticity problems in structural analysis. In Roland Glowinski, Gene H. Golub, Gérard A. Meurant, and Jacques Périaux, editors, Domain Decomposition Methods for Partial Differential Equations, SIAM, Philadelphia, 1988.

[7] Petter E. Bjørstad and Jan Mandel. On the Spectra of Sums of Orthogonal Projections with Applications to Parallel Computing. Technical Report, Department of Computer Science, University of Bergen, Thormøhlensgaten 55, N-5006 Bergen, Norway, 1989. Submitted to BIT.

[8] Petter E. Bjørstad and Olof B. Widlund. Iterative methods for the solution of elliptic problems on regions partitioned into substructures. SIAM J. Numer. Anal., 23(6):1093-1120, 1986.

[9] Petter E. Bjørstad and Olof B. Widlund. Solving elliptic problems on regions partitioned into substructures. In Garrett Birkhoff and Arthur Schoenstadt, editors, Elliptic Problem Solvers II, pages 245-256, Academic Press, New York, 1984.

[10] Petter E. Bjørstad and Olof B. Widlund. To Overlap or Not to Overlap: A Note on a Domain Decomposition Method for Elliptic Problems. SIAM J. Sci. Stat. Comput., 10(5):1053 1061, 1989.

[11] Jean-François Bourgat, Roland Glowinski, Patrick Le Tallec, and Marina Vidrascu. Variational formulation and algorithm for trace operator in domain decomposition calculations. In Tony 
Chan, Roland Glowinski, Jacques Périaux, and Olof Widlund, editors, Domain Decomposition Methods, SIAM, Philadelphia, 1988.

[12] James B. Bramble. A second order finite difference analogue of the first biharmonic boundary value problem. Numer. Math., 9:236-249, 1966.

[13] James H. Bramble, Richard E. Ewing, Joseph E. Pasciak, and Alfred H. Schatz. A preconditioning technique for the efficient solution of problems with local grid refinement. Comput. Meth. Appl. Mech. Engin., 67:149-159, 1988.

[14] James H. Bramble, Joseph E. Pasciak, and Alfred H. Schatz. The construction of preconditioners for elliptic problems by substructuring, III. Math. Comp., 51:415 - 430, 1988.

[15] James H. Bramble, Joseph E. Pasciak, and Alfred H. Schatz. The construction of preconditioners for elliptic problems by substructuring, I. Math. Comp., 47(175):103-134, 1986.

[16] James H. Bramble, Joseph E. Pasciak, and Alfred H. Schatz. The construction of preconditioners for elliptic problems by substructuring, II. Math. Comp., 49:1-16, 1987.

[17] James H. Bramble, Joseph E. Pasciak, and Alfred H. Schatz. The construction of preconditioners for elliptic problems by substructuring, IV. Math. Comp., 53:1-24, 1989.

[18] James H. Bramble, Joseph E. Pasciak, and Alfred H. Schatz. An iterative method for elliptic problems on regions partitioned into substructures. Math. Comp., 46(173):361-369, 1986.

[19] Xiao Chuan Cai. An additive schwarz algorithm for nonselfadjoint elliptic equations. 1989. Submitted to the Proceedings of the SIAM Conference on Domain Decomposition Methods, held in Houston Texas, March 20-22,1989. SIAM, Philadelphia, 1990, to appear.

[20] Xiao Chuan Cai. Some Domain Decomposstion Algorithms for Nonselfadjoint Elliptic and Parabolic Partial Differential Equations. Technical Report 461, Computer Science Department, Courant lnstitute of Mathematical Sciences, September 1989. Courant Institute doctoral dissertation.

[21] Maksymilian Dryja. An additive Schwarz algorithm for two and three- dimensional finite element elliptic problems. In Tony Chan, Roland Glowinski, Jacques Périaux, and Olof Widlund, editors, Domain Decomposition Methods, SIAM, Philadelphia, 1989.

[22] Maksymilian Dryja. An algorithm with a capacitance matrix for a variational-difference scheme. In G. I. Marchuk, editor, Variational-Difference Methods in Mathematical Physics, pages 63 - 73, USSR Academy of Sciences, Novosibirsk, 1981.

[23] Maksymilian Dryja. A finite element-capacitance method for elliptic problems on regions partitioned into subregions. Numer. Math., 44:153 - 168, 1984.

[24] Maksymilian Dryja. A method of domain decomposition for 3-D finite element problems. In Roland Glowinski, Gene H. Golub, Gérard A. Meurant, and Jacques Périaux, editors, First International Symposium on Domain Decomposition Methods for Partial Differential Equa. tions, SIAM, Philadelphia, 1988.

[25] Maksymilian Dryja, Wlodek Proskurowski, and Olof Widlund. A method of domain decomposition with crosspoints for elliptic finite element problems. In Bl. Sendov, editor, Optimal Algorithms, pages 97-111, Bulgarian Academy of Sciences, Sofia, Bulgaria, 1986.

[26] Maksymilian Dryja and Olof B. Widlund. An Additive Variant of the Schwarz Alternating Method for the Case of Many Subregions. Technical Report 339, also Ultracomputer Note 131, Department of Computer Science, Courant Institute, 1987.

[27] Maksymilian Dryja and Olof B. Widlund. On the Optimality of an Additive Iterative Refinement Method. Technical Report 442, also Ultracomputer Note 156, Department of Computer Science. Courant Institute, 1989. To appear in the proceeding of the Fourth Copper Mountain Conference on Multigrid Methods, held at Copper Mountain, Colorado, April 9 - 14, 1989, SIAM, Philadelphia, 1989.

[28] Maksymilian Dryja and Olof B. Widlund. Some Domain Decomposition Algorithms for Elliptic Problems. Technical Report 438 also Ultracomputer Note 155, Department of Computer Science, Courant Institute. 1989. To appear in the proceeding of the Conference on Iterative Methods for Large Linear Systems held in Austin, Texas, October 1988, to celebrate the Sixty-fifth Birthday of David M. Young, Jr., Academic Press, Orlando, Florida, 1989.

[29] Gene H. Golub and Charles F. Van Loan. Matrix Computations. Johns Hopkins Univ. Press, 1989. Second Edition.

[30] Anne Greenbaum, Congming Li, and Han Zheng Chao. Parallelizing Preconditioned Conjugate Gradient Algorithms. Technical Report, Courant Institute, 1988. To appear in Computer Physics Communications.

[31] Leslie Hart and Steve McCormick. Asynchronous Multilevel Adaptive Methods for Solving Partial Differential Equations on Multiprocessors: Computational Analysis. Technical Report, 
Comp. Math. Group, Univ. of Colorado at Denver, 1987. Submitted to Parallel Computing.

[32] V. I. Lebedev. Composition methods. USSR Academy of Sciences, Moscow, 1986. In Russian.

[33] Jacques Louis Lions and Enrico Magenes. Nonhomogenous Boundary Value Problems and Applications. Volume I, Springer, New York, Heidelberg, Berlin, 1972.

[34] Pierre Louis Lions. On the Schwarz alternating method. I. In Roland Glowinski, Gene H. Golub, Gérard A. Meurant, and Jacques Périaux, editors, First International Symposium on Domain Decomposition Methods for Partial Differential Equations, SIAM, Philadelphia, 1988.

[35] Jan Mandel. Hierarchical preconditioning and partial orthogonalization for the p-version finite element method. In Tony F. Chan, Roland Glowinski, Jacques Périaux, and Olof Widlund, editors, Domain Decomposition Methods, SIAM, Philadelphia, 1990. To appear in Proceedings of the Third International Symposium on Domain Decomposition Methods for Partial Differential Equations, Houston, Texas, March, 1989.

[36] Jan Mandel. Iterative solvers by substructuring for the p-version finite element method. Comput. Meth. Appl. Mech. Engin., 1989. To appear in a special issue as Proceedings of an International Conference on Spectral and High Order Methods, Como, Italy, June 1989.

[37] Jan Mandel. Two-level domain decomposition preconditioning for the p-version finite element version in three dimensions. Int. J. Numer. Meth. Engin., 1989. To a ppear.

[38] Jan Mandel and Steve McCormick. Iterative solution of elliptic equations with refinement: The two-level case. In Tony Chan, Roland Glowinski, Jacques Périaux, and Olof Widlund, editors, Domain Decomposition Methods, SIAM, Philadelphia, 1989.

[39] G. I. Marchuk, Yu. A. Kuznetsov, and A. M. Matsokin. Fictitious domain and domain decomposition methods. Soviet Journal of Numerical Analysis and Mathematical Modelling, 1:3 $61,1986$.

[40] Tarek P. Mathew. Domain Decomposition and Iterative Refinement Methods for Mixed Finite Element Discretisations of Elliptic Problems. Technical Report 463, Computer Science Department, Courant Institute of Mathematical Sciences, September 1989. Courant Institute doctoral dissertation.

[41] A. M. Matsokin and S. V. Nepomnyaschikh. A Schwarz alternating method in a subspace. Soviet Mathematics, 29(10):78 - 84, 1985.

[42] Steve McCormick. Fast adaptive composite grid (FAC) methods. In K. Bōhmer and H. J. Stetter, editors, Defect Correction Methods: Theory and Applications, pages 115-121, Computing Supplementum 5, Springer-Verlag, Wien, 1984.

[43] Steve McCormick and Jim Thomas. The fast adaptive composite grid (FAC) method for elliptic equations. Math. Comp., 46(174):439-456, 1986.

[44] S. V. Nepomnyaschikh. On the application of the method of bordering for elliptic mixed boundary value problems and on the difference norms of $W_{2}^{1 / 2}(S)$. Technical Report 106, Computing Center of the Siberian Branch of the USSR Academy of Sciences, Novosibirsk, 1984. In Russian.

[45] Alfio Quarteroni. Domain decomposition algorithms for the Stokes equations. In Tony Chan, Roland Glowinski, Jacques Périaux, and Olof Widlund, editors, Domain Decomposition Methods, SIAM, Philadelphia, 1989.

[46] P. A. Raviart and J. M. Thomas. A mixed finite element method for 2-nd order elliptic problems. In A. Dold and B. Eckmann, editors, Mathematical Aspects of Finite Element Methods, Springer, 1975. Lecture Notes of Mathematics, Volume 606.

[47] H. A. Schwarz. Gesammelete Mathematische Abhandlungen, pages 133-143. Volume 2, Springer, Berlin, 1890. First published in Vierteljahrsschrift der Naturforschenden Gesellschaft in Zürich, volume 15, 1870, pp. 272-286.

[48] Barry F. Smith. An Optimal Domain Decomposition Preconditioner for the Finite Element Solution of Linear Elasticity Problems. Technical Report 482, Department of Computer Science, Courant Institute, 1989.

[49] Barry F. Smith and Olof B. Widlund. A Domain Decomposition Algorithm Based on a Change to a Hierarchical Basis. Technical Report 473, also appeared as an Ultracomputer Note., Department of Computer Science, Courant Institute, 1989. Submitted for publication in SIAM J. Sci. Stat. Comput.

[50] S. L. Sobolev. L'Algorithme de Schwarz dans la Théorie de l'Elasticité. Comptes Rendus (Doklady) de l'Académie des Sciences de l'URSS, IV((XIII) 6):243-246, 1936.

[51] Richard S. Varga. Matrix Iterative Analysis. Prentice-Hall, 1962.

[52] Olof B. Widlund. A Comparison of Some Domain Decomposition and Iterative Refinement 
Algorithms for Elliptic Finite Element Problems. Technical Report BSC 88/15, IBM Bergen Scientific Centre, Thormøhlensgaten 55, N-5006 Bergen, Norway, 1988.

[53] Olof B. Widlund. An extension theorem for finite element spaces with three applications. In Wolfgang Hackbusch and Kristian Witsch, editors, Numerical Techniques in Continuum Mechanics, pages 110-122, Notes on Numerical Fluid Mechanics, v. 16, Friedr. Vieweg und Sohn, Braunschweig/Wiesbaden, 1987. Proceedings of the Second GAMM-Seminar, Kiel, January, 1986.

[54] Olof B. Widlund. Iterative substructuring methods: Algorithms and theory for elliptic problems in the plane. In Roland Glowinski, Gene H. Golub, Gérard A. Meurant, and Jacques Périaux, editors, First International Symposium on Domain Decomposition Methods for Partial Differential Equations, SIAM, Philadelphia, 1988.

[55] Olof B. Widlund. On the Rate of Convergence of the Classical Schwarz Alternating Method in the Case of more than Two Subregions. Technical Report, Department of Computer Science, Courant Institute, 1989. A revised version in preparation.

[56] Olof B. Widlund. Optimal iterative refinement methods. In Tony Chan, Roland Glowinski, Jacques Périaux, and Olof Widlund, editors, Domain Decomposition Methods, SIAM, Philadelphia, 1989.

[57] Jinchao Xu. Theory of Multilevel Methods. PhD thesis, Cornell University, May 1989.

[58] Harry Yserentant. On the multi-level splitting of finite element spaces. Numer. Math., 49:379$412,1986$. 


\author{
Towards a Inified Theory of Domain Decomposition Algorithms for \\ Elliptic Problems \\ by \\ Maksymilian Dryja, Olof B. W'idlund \\ Ultracomputer Note \#167 \\ Technical Report \#486
}

December 1989

\begin{abstract}
A distinction is often made between domain decomposition algorithms for elliptic partial differential cquations which use overlapping subregions and those which do not. Schwarz's alternating incthod, the oldest of them all, belongs to the first category. It has recently becn discovered that inany of the algorithms that belong to the sccond catcgory can also be regarded as generalizations of the classical Schwarz. method or an additive viriant thereof. This new approach provides new tools for the analysis and development of domain decomposition algorithms. In this paper, we introduce an abstract additive Schwarz method and develop a simple framework for the analysis of its rate of convergence. We show that it is possible and convenient 16 specily algorithms in terons of a set of subspaces and relited irthogonal projections. This family of algorithms is further cxpanded by replacing the linear systems of equations, which correspend to the projections, by suitabic preconditioners.

We illustrate the usefulness of this approach by cousidering addlitive Schwarz methods of a relatively conventional type, iterative substructuring methods, domain decomposition incthods defincd on the curves or surfaces which subdivide the region, and itcrative refincment methods. Throughout we work in a lramework of conforming finite elements and self-atloint problems, but we also mention some new results for more general elliptic finite element problems.
\end{abstract}

Key Words. Schwaris sltcrnaling method, domain decomposition, elliplic equations, finite clements.

This work was supporfed in pitt by the Nalional Science lioundasion under cirant NSli.CCR-840.3003 at 1 he Courant Mathematies and Compulugg, Laboralory and, in parl by the U.S. Department of lincrgy under contract 1)l: AC02-76ER03077-V at the Courant Nathemalics and Compuling 1 ibotatory. This report was prepaled for the procect.

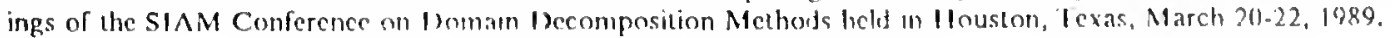



1. Introduction. In this paper, we survey our recent research on domain decomposition and related algorithms for elliptic finite element problems. One of our aims is to develop a set of common and powerful tools for the development and analysis of a great variety of methods. We have recently discovered that many iterative substructuring methods can be viewed as so-called additive Schwarz methods and that the analysis of these methods can be simplified; cf. Dryja and Widlund [28]. Here, we discuss and extend this result and provide further evidence that many domain decomposition methods fit into a framework provided by the Schwarz additive methods.

We note that the bounds given for the condition number of the iteration operators for many domain decomposition algorithms considered in this and other papers are either uniform in the number of subdomains and subregions or grow only polynomially in the logarithm of the number of degrees of freedom associated with an individual subregion. The algorithms are therefore, in a certain sense, almost optimal.

The paper is organized as follows. After introducing two elliptic model problems and certain finite element methods in Section 2, we begin Section 3 by reviewing Schwarz's alternating algorithm in its classical setting. Following Sobolev [50] and P. L. Lions [34], we indicate how this algorithm can be expressed in a variational form. Since this formulation is very convenient for the analysis of finite element problems, we work in this Hilbert space setting throughout the paper.

In Section 3, we also introduce the additive variant of Schwarz's method; cf Dryja [21], Dryja and Widlund [26], [28], Matsokin and Nepomnyaschikh [41] and Nepomnyaschikh [44]. We present it in a general form and show how algorithms of this kind can be defined in terms of a set of subspaces and projections. An additive Schwarz method can be viewed as an iterative method for the solution of an auxiliary linear problem that has the same solution as the original finite element problem. We also introduce tools, which make it possible to estimate the condition number $\kappa(P)$ of the operator $P$ of this new problem.

It is often straightforward to obtain an upper bound for the spectrum of $P$. A lower bound of the eigenvalues is obtained in terms of an upper bound of a Rayleigh quotient which measures the extent by which the subspaces are linearly independent. (If the subspaces are orthogonal, the Schwarz method converges in one step.) We show that preconditioners can be used as approximate solvers for the linear systems, which are related to the individual projections, and that it is easy to obtain bounds for the condition number of the resulting algorithm in terms of the spectrum of the basic operator and any bounds for the individual preconditioned problems that might be available.

Section 3, thus, provides us with a basic framework. In the rest of the paper, we turn to a series of applications.

In Section 4, we summarize our work on additive Schwarz methods of a type directly related to the classical case. For previous discussions of this algorithm, see Dryja [21], Dryja and Widlund [26], [28], with the most details given in [28]. We note that this method has been extended successfully to certain stationary and parabolic convection-diffusion problems in the dissertation of Xiao-Chuan Cai [20],[19], and to mixed finite element methods of the Raviart-Thomas type, cf. [46], in the dissertation of Tarek Mathew [40]. In this paper, we are unable to provide details about their work since it would require the introduction of many notations, etc. We also discuss a result on multilevel algorithms obtained in collaboration with Xuejun Zhang, a 
Courant Institute graduate student.

In Section 5, we discuss a well-known family of domain decomposition methods known as iterative substructuring methods; cf. e.g. Agoshkov [1], Bjørstad and Hvidsten [6], Bjørstad and Widlund [8], Bramble, Pasciak and Schatz [18], [15], [16], [14], [17], Dryja [24], Dryja, Proskurowski and Widlund [25], Dryja and Widlund [28], Lebedev [32], Marchuk, Kuznetsov and Matsokin [39], Quarteroni [45], Smith and Widlund [49] and Widlund [54]. These algorithms are based on a non-overlapping subdivision $\left\{\Omega_{i}\right\}$ of the region $\Omega$. ( Borrowing a term from structural engineering, the subregions are often called substructures.) Surprisingly enough, as shown already in Dryja and Widlund [28], many of these methods also fit well in the Schwarz framework. In this paper, we further extend our analysis and include a more detailed discussion of the three-dimensional case. In this study, it is fruitful to take a substructure by substructure view, estimating the contribution to the strain energy from an individual substructure in terms of the corresponding local contribution to the preconditioner. This approach is similar to that of Jan Mandel, and his coworkers Babuška, Craig and Pitkäranta, cf. [2], [37], [36], [35], who have begun a systematic study of domain decomposition methods for p-version finite element methods; $c f$. also Bramble, Pasciak and Schatz [17] for a fundamental study of h-version methods. This approach highlights how preconditioners can be constructed from parts which are strictly local with respect to an individual substructure, parts which involve interaction between pairs of neighboring substructures and a coarse global model with relatively few degrees of freedom. Many of the results which were obtained for two subregions in the early development of the theory can now be recycled to yield useful results for the much more interesting case of many substructures. We note that some of our results have been extended to a class of two-dimensional, stationary and parabolic convection-diffusion problems by Xiao-Chuan Cai [20], [19].

In Section 6, we consider Schwarz methods on the lower dimensional manifolds formed by the curves or surfaces, which partition the domain into substructures. These algorithms can also be viewed as Schwarz methods for problems of potential theory. This idea has previously been discussed by Nepomnyaschikh [44] for the case of a few substructures and without a full development of the theory. We include a discussion and estimates of the rate of convergence of a method developed by Bourgat, Glowinski, Le Tallec and Vidrascu [11] and also discuss some recent work by Barry Smith, a Courant Institute graduate student; cf. [48].

In Section 7, we survey our recent work on iterative refinement methods. These are methods for the solution of the linear systems of algebraic equations, which arise from elliptic finite element problems defined on composite meshes. The study of these methods was pioneered by McCormick and his coworkers Hart and Thomas [31], [42], [43]. An analysis of two-level algorithms is given in Bramble, Ewing, Pasciak and Schatz [13] and in Mandel and McCormick [38]. Proofs of the results discussed in this section are given in Dryja and Widlund [27] and Widlund [56]. The main difficulties in this work are related to an effort of obtaining bounds for the rate of convergence which are independent of the number of refinement levels as well as the number of degrees of freedom. We note that in the dissertation of Tarek Mathew [40], certain results are obtained on iterative refinement methods for Raviart-Thomas finite elements.

In this paper, we primarily consider algorithms that are of additive Schwarz type. We note that a variety of multiplicative algorithms also can be defined systematically. At present, the general multiplicative case appears to be less well understood than 
the additive, except in the case of two subspaces. For the two subspace case, Bjørstad and Mandel [7] have recently extended their earlier work, cf. [5] and [38], obtaining a detailed comparison of the spectra of the additive and multiplicative algorithms. We note that there are cases for which a satisfactory theory already exists in the case of more than two subspaces. Thus the algorithm of Bank, Dupont and Yserentant [3] is a multiplicative variant of the original, additive algorithm of Yserentant's [58]; it is just as well understood. Similarly, the basic theory for the multilevel iterative refinement methods has advanced to the same level in the two cases; $c f$. Widlund [56] and Dryja and Widlund [27]. We note, finally, that in the recent thesis of Mathew important progress is reported for the general multiplicative case; $c f$. Mathew [40] and a remark in Section 3.

2. Model Problems and Finite Elements. In this section, we introduce finite element approximations of a standard Poisson equation and a special second order elliptic problem with variable coefficients.

In the model problems, the continuous and discrete problems are of the form

$$
a(u, v)=f(v), \forall v \in V
$$

and

$$
a\left(u_{h}, v_{h}\right)=f\left(v_{h}\right), \forall v_{h} \in V^{h}
$$

respectively. We consider homogeneous Dirichlet problems on bounded Lipschitz regions in two or three dimensions and continuous, piecewise linear finite elements. For the Poisson problem, the bilinear form is defined by

$$
a(u, v)=\int_{\Omega} \nabla u \cdot \nabla v d x
$$

This form defines a semi-norm $|u|_{H^{1}(\Omega)}=(a(u, u))^{1 / 2}$ in the Sobolev space $H^{1}(\Omega)$. It is a norm of $V=H_{0}^{1}(\Omega)$. Here $H_{0}^{1}(\Omega)$ is the subspace of $H^{1}(\Omega)$ functions with zero trace; all elements of $V$ and its subspace $V^{h}$ vanish on $\partial \Omega$, the boundary of $\Omega$. We note that if $\tilde{\Omega} \subset \Omega$, then any element of $H_{0}^{1}(\tilde{\Omega})$ can be extended by zero to an element in $H_{0}^{1}(\Omega)$, and that the extension operator is continuous. We therefore regard $H_{0}^{1}(\tilde{\Omega})$ as a subspace of $H_{0}^{1}(\Omega)$.

Almost all our results can be extended immediately to general conforming finite element approximations of any self-adjoint elliptic problem, which can be formulated as a minimization problem, and to more general boundary conditions. However, some of the bounds can be very poor if there is a great variation in the values of the coefficients. In this paper, we consider only one generalization,

$$
a(u, v)=\int_{\Omega} \rho(x) \nabla u \cdot \nabla v d x
$$

where $\rho(x)>0$ can be discontinuous, but varies slowly in each subregion. There is experimental evidence that some domain decomposition methods of Neuman-Dirichlet type perform quite poorly for certain problems of the form (3); cf. Greenbaum, Li and Chao, [30]. For other algorithms a satisfactory theory already exists with bounds which only depend on the local variation of $\rho(x)$; see Section 5 . For still others, this issue is still to be fully addressed. 
The triangulation of $\Omega$ is introduced in the following way. The region is first divided into non-overlapping substructures $\Omega_{i}, i=1, \cdots, N$. We assume that the substructures are chosen so that the discontinuities of $\rho(x)$ occur only at substructure boundaries. To simplify the description, we confine most of our study to triangular (simplicial) substructures. In such a case, the original region must, of course, be a polygon (polyhedron). We note that the rate of convergence of the iterative methods, which are considered in this paper, depend only very mildly on the size of the substructures. The size of the subregions can therefore be selected primarily to minimize the cost of a single iteration on a particular computer system.

All the substructures $\Omega_{i}$ are further divided into elements. The common assumption in finite element theory that all elements are shape regular is adopted and the same assumption is made concerning the substructures. On the element level this means that there is a uniform bound on $h_{K} / r_{K}$, which is independent of the number of degrees of freedom. Here $h_{K}$ is the diameter of the element $K$ and $r_{K}$ the diameter of the largest inscribed sphere in $K$.

Since $a\left(u_{h}, v_{h}\right)=a\left(u, v_{h}\right), \forall v_{h} \in V^{h}$, the finite element solution is the projection of the exact solution onto the finite element space with respect to the inner product defined by the bilinear form. The problems defined on the subregions, from which preconditioners for the entire problem can be assembled, can often similarly be viewed in terms of orthogonal projections onto subspaces directly associated with the subregion in question.

We also need to use matrix representations of the finite element problems since many algorithmic details can best be described using matrix notations. When doing this, it is convenient to consider domain decomposition in light of structural engineering computational practices. The elements of the stiffness matrix $K$ are given by

$$
k_{l, m}=a\left(\varphi_{l}, \varphi_{m}\right),
$$

where $\varphi_{l}$ and $\varphi_{m}$ are standard finite element basis functions. Since an integral over $\Omega$ can be written as a sum of integrals over the substructures, the stiffness matrix can be assembled from the stiffness matrices $H^{(i)}$, which have the elements

$$
k_{l, m}^{(i)}=a_{\Omega_{1}}\left(\varphi_{l}, \varphi_{m}\right) \text {. }
$$

Here the relevant $l$ and $m$ correspond to degrees of freedom associated with the closure of the substructure $\Omega_{i}$. The bilinear form $a_{\Omega_{1}}\left(u_{h}, v_{h}\right)$ represents the contribution to the integral $a_{\Omega}\left(u_{h}, v_{h}\right)$ from the substructure $\Omega_{i}$.

This so-called subassembly process can be summarized in the formula

$$
x^{T} K^{\prime} y=\sum x^{(i)^{T}} K^{(i)} y^{(i)}
$$

where $x^{(i)}$ is the subvector of parameter values associated with $\bar{\Omega}_{i}$.

Remark. In practice, the subassembly process is often used recursively, creating larger and larger so-called super elements. This process can be interleaved with the elimination of all the variables which, at the level in question, are coupled only to other variables of the same super element; cf. e.g. [4]. Except for a brief discussion in Section 4, we consider only three levels in this paper: the elements with a characteristic diameter $h$, the substructures with a diameter on the order of $\mathrm{H}$ and the entire region $\Omega$, which, without loss of generality, is assumed to have unit diameter. 
If we divide the subvectors $x^{(i)}$ associated with the $\mathrm{i}$-th substructure into two, $x_{I}^{(i)}$ and $x_{B}^{(i)}$, corresponding to the variables which are interior to the substructure and those which are shared with other substructures, then the matrix $K^{(i)}$ can be written as

$$
\left(\begin{array}{cc}
K_{I I}^{(i)} & K_{I B}^{(i)} \\
K_{I B}^{(i) T} & K_{B B}^{(i)}
\end{array}\right)
$$

Since the interior variables are associated with only one of the substructures, they can be eliminated locally and in parallel. The reduced matrix is a so-called Schur complement and has the form

$$
S^{(i)}=K_{B B}^{(i)}-K_{I B}^{(i)}{ }^{T} K_{I I}^{(i)}{ }^{-1} K_{I B}^{(i)}
$$

It is now easy to show that if the corresponding Schur complement of the global stiffness matrix $K$ is denoted by $S$, then,

$$
x_{B}^{T} S y_{B}=\sum x_{B}^{(i)}{ }^{T} S^{(i)} y_{B}^{(i)} .
$$

The elimination of the interior variables from the substructures can be viewed in terms of orthogonal projections, with respect to the bilinear form, of the solution $u_{h}$ of equation (1) onto the subspaces $H_{0}^{1}\left(\Omega_{i}\right) \cap V^{h}, i=1, \cdots, N$. It is easy to show that these subspaces are orthogonal, in the sense of the bilinear form (4), to the so-called piecewise discrete harmonic functions given by

$$
K_{I I}^{(i)} x_{I}^{(i)}+K_{I B}^{(i)} x_{B}^{(i)}=0, \quad \forall i
$$

If the local problems are solved exactly, what remains is to find a sufficiently accurate approximation of the part of the solution which is piecewise discrete harmonic. This can be done by approximately solving the reduced linear system with the matrix $S$. A specific iterative substructuring method is obtained by selecting a preconditioner for the matrix $S$. Once an approximation of the solution has been found on the boundaries of the substructures, the solution can be found everywhere by separately solving local Dirichlet problems on each substructure.

An important family of domain decomposition algorithms can be derived by replacing each contribution $S^{(i)}$ to the Schur complement by a different matrix $\hat{S}^{(i)}$. A preconditioner $\hat{S}$ of $S$ is then created by assembling the local contributions in the same way as in equation (5). When choosing $\hat{S}$, it is important to create a linear system, which is cheaper to solve and, at the same time, to ensure that $\kappa\left(\hat{S}^{-1} S\right)$ is small. A natural idea is to eliminate the coupling between the groups of variables associated with different edges (faces) of the individual substructures. Further details are given in Dryja and Widlund [28], where this process is interpreted as a splitting in the sense of Varga [51]. In the analysis, a simple and powerful idea is based on the fact that if

$$
c_{i} x_{B}^{(i)}{ }^{T} \hat{S}^{(i)} x_{B}^{(i)} \leq x_{B}^{(i)}{ }^{T} S^{(i)} x_{B}^{(i)} \leq C_{i} x_{B}^{(i)}{ }^{T} \hat{S}^{(i)} x_{B}^{(i)}, \forall x_{B}^{(i)}
$$

then,

$$
\underline{c} x_{B}^{T} \hat{S} x_{B} \leq x_{B}^{T} S x_{B} \leq \bar{C} x_{B}^{T} \hat{S} x_{B}, \forall x_{B}
$$


where

$$
\underline{c}=\min _{i} c_{i} \text { and } \bar{C}=\max _{i} C_{i} \text {. }
$$

When this technique can be used, it is as easy to derive satisfactory bounds for equations (3) as for (2); cf. Section 5 .

3. Multiplicative and Additive Schwarz Methods. In this section, we first review Schwarz's classical alternating method [47], its variational formulation, cf. P. L. Lions [34], and an additive variant of the algorithm, cf. Dryja [21], Dryja and Widlund [26] and Matsokin and Nepomnyaschikh [41]. We then discuss a general approach to the analysis of the rate of convergence of the additive algorithms.

We begin by briefly discussing the classical formulation of Schwarz's method in the case of the continuous Poisson equation. There are two fractional steps corresponding to two overlapping subregions, $\Omega_{1}^{\prime}$ and $\Omega_{2}^{\prime}$, the union of which is the region $\Omega$. Let an initial guess $u^{0} \in V$ be given. The iterate $u^{n+1}$ is determined from $u^{n}$ by sequentially updating the approximate solution in the two subregions:

$$
\begin{aligned}
-\Delta u^{n+1 / 2} & =f & & \text { in } \Omega_{1}^{\prime}, \\
u^{n+1 / 2} & =u^{n} & & \text { on } \partial \Omega_{1}^{\prime},
\end{aligned}
$$

and

$$
\begin{aligned}
-\Delta u^{n+1} & =f & & \text { in } \Omega_{2}^{\prime}, \\
u^{n+1} & =u^{n+1 / 2} & & \text { on } \partial \Omega_{2}^{\prime} .
\end{aligned}
$$

We could just as well have written down the finite element version of the algorithm. From now on, we only consider that case. It is easy and convenient to describe this method in terms of two projections $P_{i}, i=1,2$, onto $V_{i}^{h}=H_{0}^{1}\left(\Omega_{i}^{\prime}\right) \cap V^{h} ;$ cf. Lions [34]. The projections are defined by

$$
a\left(P_{i} v_{h}, \phi_{h}\right)=a\left(v_{h}, \phi_{h}\right), \forall \phi_{h} \in V_{i}^{h}
$$

It is also easy to show that the error propagation operator of this multiplicative Schwarz method is

$$
\left(I-P_{2}\right)\left(I-P_{1}\right)
$$

This algorithm can therefore be viewed as a simple iterative method for solving

$$
\left(P_{1}+P_{2}-P_{2} P_{1}\right) u_{h}=g_{h},
$$

with an appropriate right-hand side $g_{h}$.

This algorithm can be generalized immediately to any number of subspaces. Let

$$
V^{h}=V_{1}+V_{2}+\ldots+V_{N} \text {. }
$$

The subspaces $V_{i}$ can be chosen quite arbitrarily. The projections are defined as in equation $(10)$.

The operator of (11) is a polynomial of degree two and, thus, the method is not ideal for parallel computing, since two sequential steps are involved. If more than two subspaces are used, this effect is further pronounced even if the degree of the 
polynomial representing the multiplicative algorithm often is lower than maximal. This is so because a product of two projections associated with subspaces that do not intersect nontrivially vanishes; $\mathrm{cf}$. further Widlund [52], [55]. This can best be described in terms of the coloring of an undirected graph in which the nodes represent the subspaces and the edges nontrivial intersections of pairs of subspaces. The problems associated with all subspaces of the same color can be solved in parallel since those subspaces are mutually orthogonal. For the purpose of theory, we can then regard all subspaces of the same color as one subspace; this can greatly improve the upper bound for the eigenvalues of the operator $P$, which we are about to introduce.

The basic idea behind the additive form of the algorithm is to work with the simplest possible polynomial in the projections. The equation

$$
P u_{h}=\left(P_{1}+P_{2}+\ldots+P_{N}\right) u_{h}=g_{h}^{\prime},
$$

is solved by an iterative method. (In fact, we could just as well work with $\sum_{i=1}^{N} \alpha_{i} P_{i}$, where $\alpha_{i}>0$ are suitably chosen constants.) Since we can show that the operator P is symmetric and positive definite, with respect to the bilinear form, the method of choice is the conjugate gradient method. Equation (12) must have the same solution as equation (1), i.e. the correct right-hand side must be found. Since $a\left(u_{h}, \phi_{h}\right)=f\left(\phi_{h}\right)$, by equation (1), the right-hand side $g_{h}^{\prime}$ can be constructed by solving equation (10) for all values of $i$ and adding the results. It is similarly possible to apply the operator $P$ of equation (12) to any element of $V^{h}$ by applying each projection $P_{i}$ to the element and adding the results. Most of the work, in particular that which involves the individual projections, can be carried out in parallel. We note that an additive Schwarz algorithm is fully specified by its subspaces. In this survey paper, we will sometimes just specify the subspaces without much discussion of how the corresponding algorithm can be implemented.

It is well known that the number of steps required to decrease an appropriate norm of the error of a conjugate gradient iteration by a fixed factor is proportional to $\sqrt{\kappa}$, where $\kappa$ is the condition number of the relevant operator; see e.g. Golub and Van Loan [29] . We therefore need to establish that the operator $P$ of equation (12) is not only invertible but also that satisfactory upper and lower bounds on its eigenvalues can be obtained.

An upper bound for the eigenvalues of $P$ is given by $N$ since $P$ is the sum of projections. By combining all subspaces of the same color into one, as indicated above, this bound can be improved by replacing $N$ by the number of colors of any coloring of that graph. For several algorithms this results in an upper bound, which is independent of the number of subspaces. In other instances, a useful technique is provided by strengthened Cauchy inequalities; cf. Mandel and McCormick [38], Widlund [56] and Yserentant [58].

A lower bound can often be obtained conveniently by using a lemma, inspired by Lions [34]; the simple proof is also given in Widlund [56].

LEMMA 1. Let $u_{h}=\sum_{i=1}^{N} u_{h, i}$, where $u_{h, i} \in V_{i}$, be a representation of an element of $V^{h}=V_{1}+\ldots+V_{N}$. If the representation can be chosen so that $\sum_{i=1}^{N} a\left(u_{h, i}, u_{h, i}\right) \leq$ $C_{0}^{2} a\left(u_{h}, u_{h}\right), \forall u_{h} \in V^{h}$, then $\lambda_{\min }(P) \geq C_{0}^{-2}$.

Remark. If we expand individual subspaces, there is a larger choice in selecting $u_{h, i} \in V_{i}$. The best bound for $C_{0}$ can then only improve. If we can expand the subspaces without worsening the upper bound, which is often possible, our estimate 
of $\kappa(P)$ improves. On the other hand, an enlarged subspace also means that the subproblem has more variables and that it is worse conditioned. For the special case of the classical Schwarz method on two regions, this tradeoff is well understood; for a discussion of precise estimates of the rate of convergence; see Bjorstad and Widlund $[10]$.

Remark. In his recent dissertation, Tarek Mathew [40] has given a bound on the rate of convergence of the multiplicative algorithm in terms of $C_{0}$ and the number of colors of the graph. While his bound can probably be improved considerably, it nevertheless shows that if these parameters are bounded independently of the mesh parameters, then the spectral radius of the error propagation operator is uniformly bounded by a constant that is less than 1 .

One of the attractive features of the framework introduced in this section is the ease by which variants of the basic algorithm, obtained by replacing the local linear systems by preconditioners, can be analyzed. Let us, for example, consider the additive Schwarz method for a finite element approximation of the problem discussed in the beginning of this section. We can write the projection $P_{1}$ in matrix terms. After a suitable permutation of the variables, it corresponds to

$$
y=P_{1} x=\left(\begin{array}{cc}
K_{(1)}^{-1} & 0 \\
0 & 0
\end{array}\right) K x .
$$

Here $K_{(1)}$ represents the stiffness matrix of the Dirichlet problem on $\Omega_{1}^{\prime}$. It is easy to see that the matrix of (13) is symmetric in the $K$-inner product that corresponds to the bilinear form. If $K_{(1)}^{-1}$ is replaced by $\hat{K}_{(1)}^{-1}$, etc., then it is easy to show by using a Rayleigh quotients argument that the eigenvalues of the resulting operator $\hat{P}$ satisfy

$$
\lambda_{\min }(P) \min _{i}\left(\lambda_{\min }\left(K_{(i)}^{\prime} \hat{H}_{(i)}^{-1}\right)\right) \leq \lambda_{\min }(\hat{P})
$$

and

$$
\lambda_{\max }(\hat{P}) \leq \lambda_{\max }(P) \max _{i}\left(\lambda_{\max }\left(K_{(i)} \hat{K}_{(i)}^{-1}\right)\right)
$$

An estimate of $\kappa(\hat{P})$ follows immediately,

$$
\kappa(\hat{P}) \leq \frac{\max _{i} \lambda_{\max }\left(K_{(i)}{\hat{K_{(i)}^{-1}}}^{-1}\right)}{\min _{i} \lambda_{\min }\left(\hat{K}_{(i)} \hat{K}_{(i)}^{-1}\right)} \kappa(P) .
$$

4. Additive Schwarz Methods on Overlapping Subregions. We now describe the additive Schwarz method introduced in Dryja and Widlund [26], [28]; cf. also Dryja [21]. In those papers only Poisson's equation was considered. In the original technical report, good results were given only for problems in two dimensions, but we now need no such restrictions. The most satisfactory proof, so far, is given in [28]. Here, we also discuss problems of the form (3).

We start with the same triangular (simplicial) non-overlapping substructures $\Omega_{i}$ that have been considered before. We extend each substructure to a larger region $\Omega_{i}^{\prime}$ We assume that the overlap is generous, assuming that the distance between the boundaries $\partial \Omega_{i}$ and $\partial \Omega_{i}^{\prime}$ is bounded from below by a fixed fraction of $H_{i}$, the diameter of $\Omega_{s}$. We also assume that $\partial \Omega_{i}^{\prime}$ does not cut through any element. We make the same 
construction for the substructures that are next to the boundary except that we cut off the part of $\Omega_{i}^{\prime}$ that is outside of $\Omega$.

Remark. The analysis of Schwarz methods is more complicated when the overlap is less generous; cf. a discussion in Lions [34]. Such a situation occurs if the region is L-shaped and partitioned into two overlapping rectangles. A similar situation is discussed in Dryja and Widlund [28] in an analysis of iterative substructuring methods; cf. also Section 5 .

Our finite element space is represented as the sum of $N+1$ subspaces

$$
V^{h}=V_{0}^{h}+V_{1}^{h}+\ldots+V_{N}^{h}
$$

The first subspace $V_{0}^{h}$ is equal to $V^{H}$, the space of continuous, piecewise linear functions on the coarse mesh defined by the substructures $\Omega_{i}$. The other subspaces are related to the subdomains in the same manner as in a traditional Schwarz algorithm, i.e. $V_{i}^{h}=H_{0}^{1}\left(\Omega_{i}^{\prime}\right) \cap V^{h}$.

Remark. There are few differences between the problem related to the first subspace and the others. The right hand side of the particular linear system is generated as weighted averages with weights determined by the coarse mesh basis functions. We also need to interpolate the solutions in $V_{0}^{h}$ when combining them with the contributions from the other subspaces. The coarse, global approximation of the elliptic equation is otherwise quite similar to the local problems.

The global coarse problem provides a mechanism for the global transportation of information. As shown in Widlund [54], the rate of convergence of any domain decomposition method, for which the interaction is only through next neighboring subdomains, has a condition number which grows at least as fast as $1 / H^{2}$.

The following result is established in Dryja and Widlund [28].

THEOREM 1. The operator $P$ of the additive algorithm defined by the spaces $V^{H}$ and $V_{i}^{h}$, applied to equation (2), satisfies the estimate $\kappa(P) \leq$ const.

In the proof, a quasi-interpolant, $\hat{I}_{H}$, which is a bounded operator in $H_{0}^{1}(\Omega)$ and satifies

$$
\left\|u_{h}-\hat{I}_{H} u_{h}\right\|_{L_{2}(\Omega)} \leq \text { const. } H\left|u_{h}\right|_{H^{1}(\Omega)}
$$

is used. By examining the proof, it is easy to see that if uniform bounds of the same form hold for the weighted spaces $L_{2, \rho}(\Omega)$ and $H_{0, \rho}^{1}(\Omega)$, defined by

$$
\int_{\Omega} \rho(x)|u|^{2} d x \quad \text { and } \quad \int_{\Omega} \rho(x)|\nabla u|^{2} d x
$$

respectively, then the optimality also holds for the variable coefficient case (3). This is a difficult problem, still not fully understood; cf. $\mathrm{Xu}_{\mathbf{u}}[57]$ for partial results. We note that for two-dimensional problems the proof given in Dryja and Widlund [26] works just as well for equation (3). The bound of the condition number given in that paper is proportional to $(1+\log (H / h))$.

We have also considered the use of more than two levels of partitioning of the region and established that for equation $(2) \kappa(P)$ does not grow faster than quadratically in the number of levels. We do not know if this result can be improved. For convex regions, we have shown that the growth is linear in the number of levels. This result cannot be improved. Our work has been carried out jointly with Xuejun Zhang, who 
has also made a large number of numerical experiments. Rapid convergence can be obtained when using such methods solving only very small linear systems of equations. As we have already pointed out in the introduction, Xiao-Chuan Cai [20], [19] and Tarek Matew [40] have extended our analysis to certain nonsymmetric and indefinite problems.

5. Iterative Substructuring Methods as Schwarz Methods. In this section, we show how iterative substructuring methods can be derived by using the framework of subspaces and projections developed in Section 3. We first consider problems in the plane and give an estimate of the condition number of the operator $P$ that corresponds to a specific choice of subspaces. For this basic algorithm, the proof of the estimate does not rely on the extension theorems, which have played an important role in previous estimates for iterative substructuring methods; cf. Widlund [53]. We then indicate how results for the special case of two substructures, and an argument about preconditioners for the subspace problems, can be used to derive different algorithms. including one due to Bramble, Pasciak and Schatz [15]. We also consider a second choice of subspaces and show that the resulting algorithm extends to a fast method for the three-dimensional case. The resulting algorithm is similar but not identical to one of the two algorithms considered in Bramble, Pasciak and Schatz [17]; cf. also Dryja [24] for early work on the three-dimensional case. The discussion in this section extends that in Dryja and Widlund [28] and relies in part on ideas from Mandel [36].

We assume that the region is divided into substructures and elements as in Section 2. We first consider the two-dimensional case and introduce an additive Schwarz method. We use the coarse space $V^{H}$ introduced in Section 4 and let the subregions $\Omega_{i j}=\Omega_{i} \cup \Gamma_{i j} \cup \Omega_{j}$ play the same role as $\Omega_{i}^{\prime}$ in Section 4 . Here $\Omega_{i}$ and $\Omega_{j}$ are adjacent substructures with a common edge $\Gamma_{i j}$. The local subspaces $V_{i j}^{h}$ are thus defined as $V_{i j}^{h}=H_{0}^{1}\left(\Omega_{i j}\right) \cap V^{h}$.

Compared with the subspaces used in the previous section, we use less overlap in the sense that only the elements of $V^{H}$ can differ from zero at the vertices of the substructures. This is reflected in a poorer bound on the condition number when we work with Lemma 1.

THEOREM 2. In the two-dimensional case, the operator $P$ of the additive algorithm defined by the spaces $V^{H}$ and $V_{i j}^{h}$ satisfies the estimate $\kappa(P) \leq$ const. $(1+\log (H / h))^{2}$ for equations (?) and (3). The constant is independent of $h, H$ and the discontinuities of the coefficient $\rho(x)$.

For this algorithm, $\lambda_{\max }(P) \leq 4$; cf. Dryja and Widlund [28]. It is easy to see that no more than four colors are needed to color the graph; each interior substructure is covered exactly three times by the subspaces associated with its three edges and, in addition, we have the global space $V^{H}$ which intersects all other subspaces. In our proof of the lower bound of the spectrum of $P$, given fully in [28], we use Lemma 1 and the following lemma, which also plays an important role in the more traditional theory of iterative substructuring algorithms.

LEMMA 2. Let $\alpha$ be any convex combination of values of $u_{h}(x), x \in \bar{\Omega}_{i}$. Then

$$
\left\|u_{h}-\alpha\right\|_{L^{\infty}\left(\Omega_{i}\right)}^{2} \leq \text { const. }(1+\log (H / h))\left|u_{h}\right|_{H^{1}\left(\Omega_{i}\right)}^{2} .
$$

